\title{
Total Synthesis of (+)-Amphidinolide A. Structure Elucidation and Completion of the Synthesis
}

Barry M. Trost,* Paul E. Harrington, John D. Chisholm, and Stephen T. Wrobleski Department of Chemistry, Stanford University, Stanford, California 94305-5080
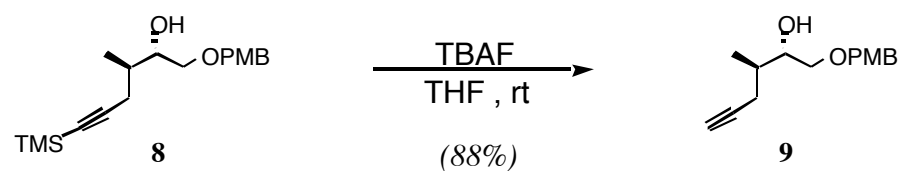

To a solution of $10.45 \mathrm{~g}(36.8 \mathrm{mmol})$ of $\mathbf{8}$ in $80 \mathrm{~mL}$ of THF at $\mathrm{rt}$ was added $40.5 \mathrm{~mL}$ (40.5 mmol) of a $1.0 \mathrm{M}$ solution of tetrabutylammonium fluoride in THF followed by stirring at rt for 45 minutes. The reaction mixture was then partitioned between saturated aqueous ammonium chloride $(50 \mathrm{~mL})$, water $(50 \mathrm{~mL})$, and diethyl ether $(50 \mathrm{~mL})$ and the resulting aqueous portion was extracted with additional diethyl ether $(3 \times 50 \mathrm{~mL})$. The combined organic extracts were washed with brine $(50 \mathrm{~mL})$, dried over anhydrous magnesium sulfate, filtered, and concentrated in vacuo to afford $9.5 \mathrm{~g}$ of the crude product mixture as a yellow oil. Flash chromatography on silica gel (elution with 50\% diethyl ether in petroleum ether) yielded $8.04 \mathrm{~g}$ $(88 \%)$ of pure 9 as a yellow oil. $\mathrm{TLC} \mathrm{R}_{\mathrm{f}}=0.43\left(50 \% \mathrm{Et}_{2} \mathrm{O}\right.$ in petroleum ether). IR (film): 3452.8, 3292.3, 2908.7, 2115.1, 1612.3, 1586.0, 1513.5, 1247.5, 1092.2, 1034.0, 818.7 $\mathrm{cm}^{-1} .{ }^{1} \mathrm{H}$ NMR (300 MHz, $\left.\mathrm{CDCl}_{3}\right)$ d: $7.26(\mathrm{~d}, J=8.3 \mathrm{~Hz}, 2 \mathrm{H}), 6.89(\mathrm{~d}, J=8.8 \mathrm{~Hz}, 2 \mathrm{H}), 4.48$ (s, $\left.2 \mathrm{H}\right)$, 3.90-3.84 (m, $1 \mathrm{H}), 3.81$ (s, $3 \mathrm{H}), 3.51(\mathrm{dd}, J=9.3,3.4 \mathrm{~Hz}, 1 \mathrm{H}), 3.42$ (dd, $J=9.3,7.8 \mathrm{~Hz}, 1 \mathrm{H})$, 2.49 (br s, $1 \mathrm{H}$ ), 2.32 (ddd, $J=16.6,6.8,2.9 \mathrm{~Hz}, 1 \mathrm{H}), 2.15$ (ddd, $J=16.6,6.8,2.9 \mathrm{~Hz}, 1 \mathrm{H}), 1.98$ $(\mathrm{t}, J=2.9 \mathrm{~Hz}, 1 \mathrm{H}), 1.87-1.79(\mathrm{~m}, 1 \mathrm{H}), 1.02(\mathrm{~d}, J=6.8 \mathrm{~Hz}, 3 \mathrm{H}) .{ }^{13} \mathrm{C} \mathrm{NMR}\left(75 \mathrm{MHz}, \mathrm{CDCl}_{3}\right)$

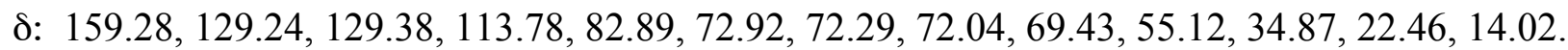
Anal. Calcd for $\mathrm{C}_{15} \mathrm{H}_{20} \mathrm{O}_{3}$ : C, 71.77; H, 7.74. Found: C, 71.68; H, 7.86. $[\alpha]_{D}^{26}=+11.1(\mathrm{c}$ $\left.1.09, \mathrm{CH}_{2} \mathrm{Cl}_{2}\right)$.
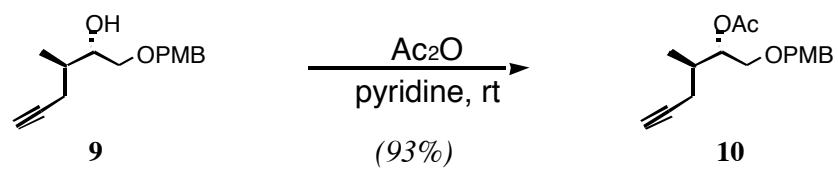

To a solution of $1.10 \mathrm{~g}(4.43 \mathrm{mmol})$ of 9 in $4.5 \mathrm{~mL}$ of pyridine at $\mathrm{rt}$ was added $2.51 \mathrm{~mL}$ (2.71 g, $26.6 \mathrm{mmol})$ of acetic anhydride and the resulting mixture was stirred at $\mathrm{rt}$ for $6 \mathrm{~h}$ then the 
mixture was poured into $80 \mathrm{~mL}$ of diethyl ether and the solution was washed with $5 \%$ aqueous $\mathrm{HCl}(4 \times 30 \mathrm{~mL})$. The combined aqueous washes were extracted with additional diethyl ether $(20 \mathrm{~mL})$ and the combined organic extracts were washed with saturated aqueous copper sulfate solution $(20 \mathrm{~mL})$, brine $(20 \mathrm{~mL})$, then dried over anhydrous magnesium sulfate, filtered, and concentrated in vacuo to afford a clear, colorless liquid as the crude product mixture. Flash chromatography on silica gel (25\% diethyl ether in petroleum ether) afforded $1.20 \mathrm{~g} \mathrm{(93 \% )} \mathrm{of} \mathrm{a}$ colorless oil as pure 10. TLC $\mathrm{Rf}_{\mathrm{f}}=0.32\left(25 \% \mathrm{Et}_{2} \mathrm{O}\right.$ in petroleum ether). IR (film): 3289.9, 2938.4, 2838.1, 2117.3, 1737.9, 1612.9, 1514.0, 1243.6, 1034.0, 820.6 $\mathrm{cm}^{-1}$. ${ }^{1} \mathrm{H}$ NMR (300 $\left.\mathrm{MHz} \mathrm{CDCl}_{3}\right)$ d: $7.22(\mathrm{~d}, J=8.8 \mathrm{~Hz}, 2 \mathrm{H}), 6.85(\mathrm{~d}, J=8.8 \mathrm{~Hz}, 2 \mathrm{H}), 5.07$ (apparent q, $J=4.9$ $\mathrm{Hz}, 1 \mathrm{H}), 4.43\left(\mathrm{AB} \mathrm{q}, v_{\mathrm{A}}=4.46, v_{\mathrm{B}}=4.40, J=11.7 \mathrm{~Hz}, 2 \mathrm{H}\right), 3.78(\mathrm{~s}, 3 \mathrm{H}), 3.54(\mathrm{dd}, J=10.7$, $5.4 \mathrm{~Hz}, 1 \mathrm{H}), 3.48(\mathrm{dd}, J=10.7,4.9 \mathrm{~Hz}, 1 \mathrm{H}), 2.28-2.07(\mathrm{~m}, 3 \mathrm{H}), 2.05(\mathrm{~s}, 3 \mathrm{H}), 1.96(\mathrm{t}, J=2.4$ $\mathrm{Hz}, 1 \mathrm{H}), 1.00(\mathrm{~d}, J=6.4 \mathrm{~Hz}, 3 \mathrm{H}) .{ }^{13} \mathrm{C} \mathrm{NMR}\left(75 \mathrm{MHz}, \mathrm{CDCl}_{3}\right) \delta: 170.53,159.16,129.89$, $129.27,113.69,81.95,74.36,72.65,69.83,68.60,55.17,32.99,22.33,21.02$, 14.45. Anal. Calcd for $\mathrm{C}_{17} \mathrm{H}_{22} \mathrm{O}_{4}: \mathrm{C}, 70.32, \mathrm{H}, 7.64$. Found: C, 70.49; H, 7.46. $[\alpha]_{\mathrm{D}}^{26}=-5.04$ (c 1.80, $\mathrm{CH}_{2} \mathrm{Cl}_{2}$ ).
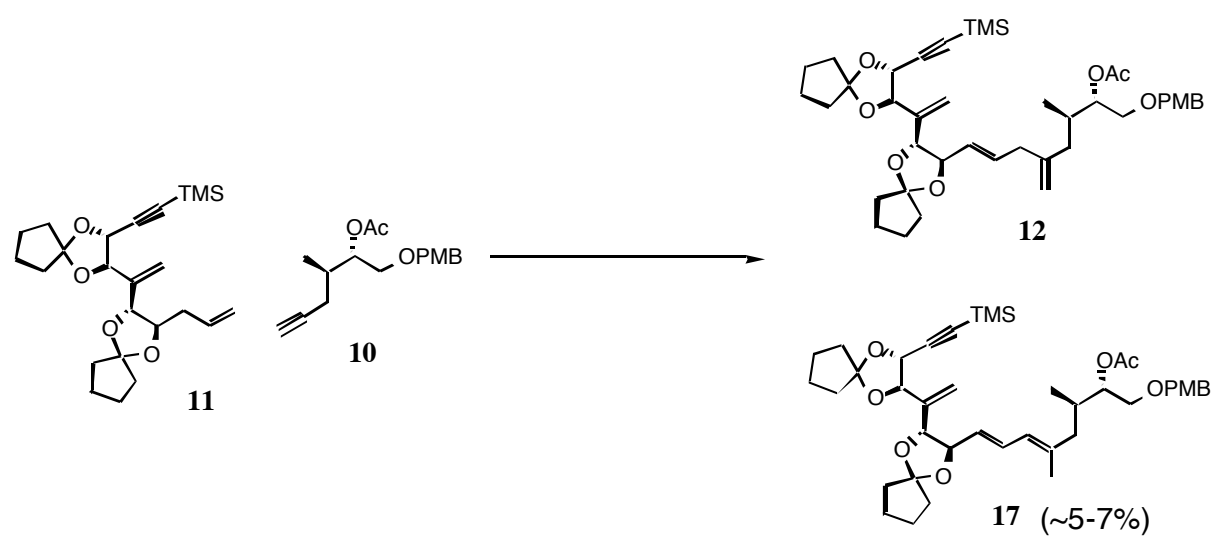

A solution of $0.354 \mathrm{~g}(0.850 \mathrm{mmol})$ of alkene 11 and $0.271 \mathrm{~g}(0.935 \mathrm{mmol})$ of alkyne 10 in $1.7 \mathrm{~mL}$ of anhydrous DMF was degassed by bubbling argon directly through the solution for 20 minutes. The degassed solution of the substrates was then rapidly transferred via cannula into a reaction tube which contained $0.037 \mathrm{~g}(0.085 \mathrm{mmol})$ of $\left[\mathrm{CpRu}\left(\mathrm{NCCH}_{3}\right)_{3}\right]^{+} \mathrm{PF}_{6}{ }^{-}$and a magnetic stir bar under an argon atmosphere. After the addition was complete, the mixture was stirred briefly (10-15 sec) at rt to give a homogeneous, dark red/brown solution, then the reaction was 
immediately placed into a preheated $60^{\circ} \mathrm{C}$ oil bath so that the top level of the reaction mixture was completely submerged below the surface of the oil bath to ensure accurate heating of the reaction mixture. During the initial heating period (within 5 minutes), the solution lightened in color to provide a medium yellow/orange solution which was heated at $60^{\circ} \mathrm{C}$ for a total reaction time of 3 hours. At this time, the reaction tube was removed from the oil bath and allowed to cool to rt, then the reaction mixture was diluted with $20 \mathrm{~mL}$ of ether and the solution was washed with water $(4 \times 3 \mathrm{~mL})$. The combined aqueous washes were extracted with diethyl ether $(3 \times 5$ $\mathrm{mL})$ and the combined organic extracts were washed with brine $(5 \mathrm{~mL})$, dried over anhydrous magnesium sulfate, filtered, and concentrated in vacuo to provide $0.640 \mathrm{~g}$ of a yellow oil as the crude product mixture. In order to efficiently separate the desired product from the unreacted alkyne 10, the crude product mixture was flash chromatographed on silica gel using a methanol/methylene chloride solvent mixture as the eluant. Initially eluting with a mixture of methanol in methylene chloride (30 pippette drops of methanol in $100 \mathrm{~mL}$ of methylene chloride) afforded $0.177 \mathrm{~g}(50 \%)$ of recovered, unreacted alkene substrate 11. Later fractions afforded $0.097 \mathrm{~g}(36 \%)$ of recovered, unreacted alkyne substrate 10. Finally, increasing the eluant polarity (50 pipette drops of methanol in $100 \mathrm{~mL}$ of methylene chloride) then provided $0.288 \mathrm{~g}(48 \%, 96 \%$ based on recovered 11) of the desired Alder ene adduct 12 which was contaminated with approximately $5-7 \%$ of an inseparable byproduct believed to be the isomerized product 17. Physical data for 12: TLC $\mathrm{R}_{\mathrm{f}}=0.13$ (30 drops of methanol in $100 \mathrm{~mL}$ of methylene chloride). IR (film): 3289.9, 2938.4, 2838.1, 2117.3, 1737.9, 1612.9, 1514.0, 1243.6, 1034.0, 820.6 cm-1. ${ }^{1} \mathrm{H}$ NMR $\left(300 \mathrm{MHz} \mathrm{CDCl}_{3}\right) \delta: 7.22(\mathrm{~d}, J=8.8 \mathrm{~Hz}, 2 \mathrm{H}), 6.85(\mathrm{~d}, J$ $=8.8 \mathrm{~Hz}, 2 \mathrm{H}), 5.07$ (apparent q, $J=4.9 \mathrm{~Hz}, 1 \mathrm{H}), 4.43\left(\mathrm{AB} \mathrm{q}, v_{\mathrm{A}}=4.46, v_{\mathrm{B}}=4.40, J_{\mathrm{AB}}=11.7\right.$ Hz, 2 H), 3.78 (s, 3 H), 3.54 (dd, $J=10.7,5.4$ Hz, $1 \mathrm{H}), 3.48$ (dd, $J=10.7,4.9 \mathrm{~Hz}, 1 \mathrm{H}), 2.28-$ $2.07(\mathrm{~m}, 3 \mathrm{H}), 2.05(\mathrm{~s}, 3 \mathrm{H}), 1.96(\mathrm{t}, J=2.4 \mathrm{~Hz}, 1 \mathrm{H}), 1.00(\mathrm{~d}, J=6.4 \mathrm{~Hz}, 3 \mathrm{H}) .{ }^{13} \mathrm{C}$ NMR $(75$ $\mathrm{MHz}_{\mathrm{CDCl}} \mathrm{CD}_{\text {) }}$ : $170.53,159.16,129.89,129.27,113.69,81.95,74.36,72.65,69.83,68.60$, 55.17, 32.99, 22.33, 21.02, 14.45. Anal. Calcd for $\mathrm{C}_{17} \mathrm{H}_{22} \mathrm{O}_{4}: \mathrm{C}, 70.32 ; \mathrm{H}, 7.64$. Found: C, 70.49; H, 7.46. $[\alpha]_{\mathrm{D}}^{24}=-5.04\left(\mathrm{c} 1.8, \mathrm{CH}_{2} \mathrm{Cl}_{2}\right)$. 


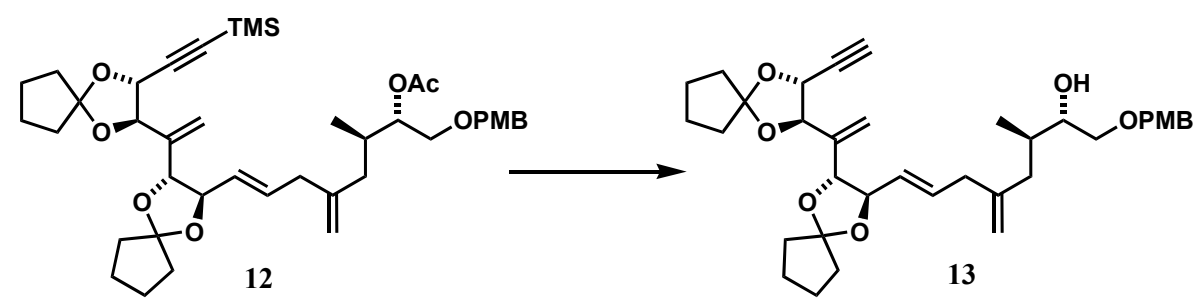

To a solution of $0.275 \mathrm{~g}(0.389 \mathrm{mmol})$ of 12 in $1.3 \mathrm{~mL}$ of methanol at $\mathrm{rt}$ was added $0.20 \mathrm{~g}$ $(1.17 \mathrm{mmol})$ of barium hydroxide and the resulting heterogeneous mixture was stirred at $\mathrm{rt}$ for $1.5 \mathrm{~h}$. The reaction mixture was concentrated on a rotary evaporator and the resulting residue was suspended in $5 \mathrm{~mL}$ of diethyl ether and the mixture was filtered through a plug of celite to remove the solids. The celite plug was washed with $10 \mathrm{~mL}$ of additional diethyl ether and the clear filtrate was concentrated in vacuo to afford $0.275 \mathrm{~g}$ of a clear, colorless oil as the crude product mixture. Flash chromatography on silica gel (45\% diethyl ether in petroleum ether) yielded $0.219 \mathrm{~g}(95 \%)$ of 13 as a colorless oil. Physical data for 13: TLC $\mathrm{R}_{\mathrm{f}}=0.35(50 \%$ diethyl ether in petroleum ether). IR (film): 3492.2, 3285.3, 2961.4, 2874.2, 2116.2, 1644.1, $1612.8,1513.9,1248.6,1104.8,1035.9,820.6 \mathrm{~cm}^{-1}$. ${ }^{1} \mathrm{H}$ NMR $\left(300 \mathrm{MHz}, \mathrm{CDCl}_{3}\right) \delta: 7.22(\mathrm{~d}, J=$ $8.3 \mathrm{~Hz}, 2 \mathrm{H}), 6.82$ (d, $J=8.3 \mathrm{~Hz}, 2 \mathrm{H}), 5.80$ (dt, $J=15.6,6.8 \mathrm{~Hz}, 1 \mathrm{H}), 5.50-5.43(\mathrm{~m}, 1 \mathrm{H}), 5.45$ (overlapping s, 1 H), 5.39 (s, 1 H), 4.75 (br s, 1 H), 4.48-4.37 (m, 4 H), 4.26-4.13 (m, 2 H), 3.76 $(\mathrm{s}, 3 \mathrm{H}), 3.70-3.65(\mathrm{~m}, 1 \mathrm{H}), 3.45-3.33(\mathrm{~m}, 2 \mathrm{H}), 2.70(\mathrm{~d}, J=6.8 \mathrm{~Hz}, 2 \mathrm{H}), 2.55(J=1.5 \mathrm{~Hz}, 1 \mathrm{H})$, 2.33 (br s, $1 \mathrm{H}), 2.16$ (dd, $J=13.7,5.4 \mathrm{~Hz}, 1 \mathrm{H}), 1.96-1.77$ (m, $10 \mathrm{H}), 1.75-1.64(\mathrm{~m}, 8 \mathrm{H}), 0.83$ $(\mathrm{d}, J=6.3 \mathrm{~Hz}, 3 \mathrm{H}) .{ }^{13} \mathrm{C} \mathrm{NMR}\left(75 \mathrm{MHz}, \mathrm{CDCl}_{3}\right) \delta: 159.74,145.45,141.42,134.37,129.95$, 129.31, 128.00, 120.28, 118.87, 115.91, 113.74, 112.53, 82.03, 81.01, 80.74, 77.50, 77.08, 76.66, $75.24,72.94,72.63,72.58,70.43,55.16,39.88,38.62,37.33,37.18,36.58,33.27,23.74,23.38$, 23.11, 13.92. $[\alpha]_{\mathrm{D}}^{24}=+23.7\left(\mathrm{c} 0.87, \mathrm{CH}_{2} \mathrm{Cl}_{2}\right)$. HRMS (EI): $m / z$ Calcd for $\mathrm{C}_{31} \mathrm{H}_{39} \mathrm{O}_{5}\left(\mathrm{M}^{+}\right.$$\left.\mathrm{C}_{5} \mathrm{H}_{8}-\mathrm{H}_{2} \mathrm{O}\right):$ 491.2798. Found: 491.2727.

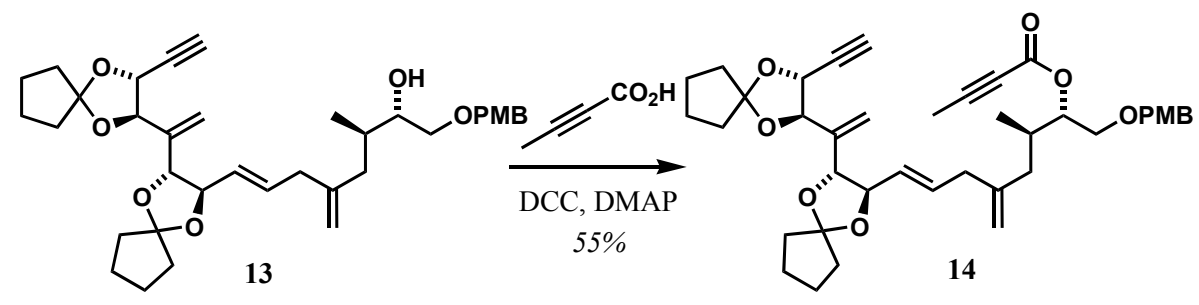

To a mixture of $0.215 \mathrm{~g}(0.363 \mathrm{mmol})$ of alcohol 13 and $0.061 \mathrm{~g}(0.726 \mathrm{mmol})$ of $2-$ butynoic acid in $0.7 \mathrm{~mL}$ of methylene chloride at $\mathrm{rt}$ was added dropwise a solution of $0.165 \mathrm{~g}$ 
( $0.799 \mathrm{mmol})$ of DCC and $0.011 \mathrm{~g}(0.73 \mathrm{mmol})$ of 4-pyrrolidinopyridine (PPY) in $0.7 \mathrm{~mL}$ of methylene chloride and the resulting yellow-orange heterogeneous mixture was stirred at $\mathrm{rt}$ for $24 \mathrm{~h}$. At this time, the mixture was diluted with $5 \mathrm{~mL}$ of methylene chloride and filtered through a plug of celite to remove the solids. The filtrate was concentrated in vacuo to afford $0.499 \mathrm{~g}$ of a reddish brown oil as the crude product mixture. Flash chromatography on silica gel (30\% diethyl ether in petroleum ether) afforded $0.166 \mathrm{~g}(57 \%)$ of $\mathbf{1 4}$ as a yellow oil. TLC $\mathrm{R}_{\mathrm{f}}=0.38$ (3\% diethyl ether in benzene). IR (film): 3280.5, 2964.6, 2874.4, 2242.5, 1709.5, 1646.1, 1612.6, 1513.9, 1252.6, 1105.9, 1065.9, $1035.7 \mathrm{~cm}^{-1} .{ }^{1} \mathrm{H}$ NMR $\left(300 \mathrm{MHz}, \mathrm{CDCl}_{3}\right) \delta: 7.20(\mathrm{~d}, J=8.8$ Hz, 2 H), 6.84 (d, $J=8.8 \mathrm{~Hz}, 2 \mathrm{H}), 5.78$ (dt, $J=15.1,6.8 \mathrm{~Hz}, 1 \mathrm{H}), 5.50-5.42(\mathrm{~m}, 1 \mathrm{H}), 5.45$ (overlapping s, $1 \mathrm{H}), 5.40(\mathrm{~s}, 1 \mathrm{H}), 5.07-5.02(\mathrm{~m}, 1 \mathrm{H}), 4.78(\mathrm{~s}, 1 \mathrm{H}), 4.74(\mathrm{~s}, 1 \mathrm{H}), 4.47-4.38(\mathrm{~m}$, $4 \mathrm{H}), 4.24$ (apparent t, $J=8.3 \mathrm{~Hz}, 1 \mathrm{H}), 4.14$ (d, $J=8.3 \mathrm{~Hz}, 1 \mathrm{H}), 3.77$ (s, $3 \mathrm{H}), 3.55-3.44$ (m, 2 H), 2.73-2.67 (m, 2 H), 2.54 (d, $J=2.0 \mathrm{~Hz}, 1 \mathrm{H}), 2.12-1.93$ (m, $3 \mathrm{H}), 1.96$ (overlapping s, $3 \mathrm{H}$ ), 1.91-1.75 (m, $8 \mathrm{H}), 1.73-1.65(\mathrm{~m}, 8 \mathrm{H}), 0.85(\mathrm{~d}, J=6.3 \mathrm{~Hz}, 3 \mathrm{H}) .{ }^{13} \mathrm{C} \mathrm{NMR}\left(75 \mathrm{MHz}, \mathrm{CDCl}_{3}\right)$

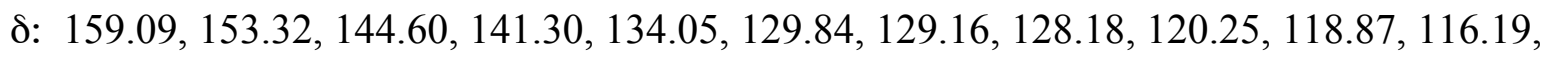
$113.66,112.98,85.77,81.87,81.17,81.00,80.70,76.58,75.16,72.63,72.39,70.38,68.51$, $55.14,39.58,38.43,37.33,37.17,36.58,31.55,23.74,23.37,23.09,14.05,3.79 .[\alpha]_{D}^{24}=+23.7$ (c $0.87, \mathrm{CH}_{2} \mathrm{Cl}_{2}$ ).
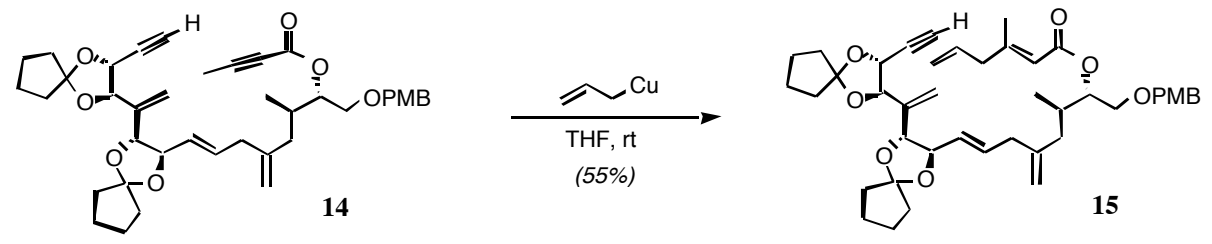

To a solution of $0.407 \mathrm{~g}(1.23 \mathrm{mmol})$ of allyltributylstannane in $3 \mathrm{~mL}$ of THF at $-78^{\circ} \mathrm{C}$ was added $0.662 \mathrm{~mL}(1.02 \mathrm{mmol})$ of a $1.54 \mathrm{M}$ solution of $n$ butyllithium in hexanes and the resulting clear, pale yellow solution was stirred at $-78^{\circ} \mathrm{C}$ for $1 \mathrm{~h}$. The solution was then transferred via cannula into a slurry of $0.093 \mathrm{~g}(1.04 \mathrm{mmol})$ of copper cyanide in $1 \mathrm{~mL}$ of THF at $-60^{\circ} \mathrm{C}$. After the addition was complete, the $-60^{\circ} \mathrm{C}$ bath was replaced with a $-25^{\circ} \mathrm{C}$ bath and the reaction mixture was allowed to stir at this temperature for $1 \mathrm{~h}$ during which time the mixture became nearly homogeneous and dark brown in color. The solution of the allylcopper reagent was cooled to $-78^{\circ} \mathrm{C}$ whereupon a solution of $0.135 \mathrm{~g}(0.205 \mathrm{mmol})$ of substrate $14 \mathrm{in} 1 \mathrm{~mL}$ of THF was added via cannula $\left(0.2 \mathrm{~mL}\right.$ THF rinse). The reaction mixture was stirred at $-78^{\circ} \mathrm{C}$ for 
$3.5 \mathrm{~h}$, then quenched at $-78^{\circ} \mathrm{C}$ by the addition of $0.2 \mathrm{~mL}$ of methanol followed by the addition of $1 \mathrm{~mL}$ of saturated aqueous ammonium chloride then the mixture was allowed to warm to rt. After stirring at $\mathrm{rt}$ for $1 \mathrm{~h}$, the contents were partitioned between $10 \mathrm{~mL}$ of water and $10 \mathrm{~mL}$ of diethyl ether and the aqueous portion was extracted with additional diethyl ether $(3 \times 10 \mathrm{~mL})$. The combined organic extracts were washed with brine $(5 \mathrm{~mL})$, dried over anhydrous magnesium sulfate, filtered, and concentrated in vacuo to afford approximately $0.5 \mathrm{~g}$ of a colorless oil as the crude product mixture. Flash chromatography on silica gel initially eluting with $20 \%$ diethyl ether in petroleum ether mixtures afforded $0.079 \mathrm{~g} \mathrm{(55 \% )}$ of $\mathbf{1 5}$ as a colorless oil. Increasing the eluant concentration to $35 \%$ diethyl ether in petroleum ether then afforded $0.042 \mathrm{~g} \mathrm{(32 \% )}$ ) of unreacted substrate 14. Yield of product based on recovered starting material is $80 \%$. Physical data for 15 : TLC $\mathrm{R}_{\mathrm{f}}=0.32$ ( $20 \%$ diethyl ether in petroleum ether). IR (film): 3279.8, 2963.1, 2874.4, 2116.2, 1715.1, 1648.8, 1613.0, 1513.9, 1334.6, 1106.3, 1036.2, 822.1 $\mathrm{cm}^{-1} .{ }^{1} \mathrm{H}$ NMR (300 MHz, $\left.\mathrm{CDCl}_{3}\right) \delta: 7.20(\mathrm{~d}, J=8.8 \mathrm{~Hz}, 2 \mathrm{H}), 6.84(\mathrm{~d}, J=8.8 \mathrm{~Hz}, 2 \mathrm{H}), 5.83-$ $5.72(\mathrm{~m}, 2 \mathrm{H}), 5.69(\mathrm{~s}, 1 \mathrm{H}), 5.50-5.42(\mathrm{~m}, 1 \mathrm{H}), 5.46$ (overlapping s, $1 \mathrm{H}), 5.40(\mathrm{~s}, 1 \mathrm{H}), 5.11(\mathrm{~d}$, $J=9.8 \mathrm{~Hz}, 1 \mathrm{H}$ ), 5.09 (overlapping d, $J=17.1 \mathrm{~Hz}, 1 \mathrm{H}$ ), 5.04-4.99 (m, $1 \mathrm{H}), 4.77$ (s, $1 \mathrm{H}$ ), 4.73 (s, $1 \mathrm{H}), 4.48-4.38$ (m, $4 \mathrm{H}$ ), 4.24 (apparent t, $J=8.3 \mathrm{~Hz}, 1 \mathrm{H}$ ), 4.14 (d, $J=8.3 \mathrm{~Hz}, 1 \mathrm{H}), 3.77$ (s, $3 \mathrm{H}), 3.55-3.43$ (m, $2 \mathrm{H}), 2.86$ (d, $J=6.8 \mathrm{~Hz}, 2 \mathrm{H}), 2.77-2.65$ (m, $2 \mathrm{H}), 2.54$ (d, $J=2.0 \mathrm{~Hz}, 1 \mathrm{H}$ ), 2.13 (s, $3 \mathrm{H}), 2.07-1.53(\mathrm{~m}, 19 \mathrm{H}), 0.82(\mathrm{~d}, J=7.8 \mathrm{~Hz}, 3 \mathrm{H}) .{ }^{13} \mathrm{C} \mathrm{NMR}\left(75 \mathrm{MHz}, \mathrm{CDCl}_{3}\right)$

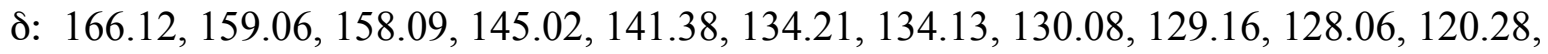
$118.89,117.84,116.25,116.09,113.65,112.69,81.97,81.14,81.01,80.74,75.17,73.83,72.58$, $70.41,68.85,55.16,44.95,39.90,38.43,37.34,37.20,36.60,31.54,23.75,23.40 .[\alpha]_{\mathrm{D}}^{24}=+$ 19.4 (c $0.81, \mathrm{CH}_{2} \mathrm{Cl}_{2}$ ).

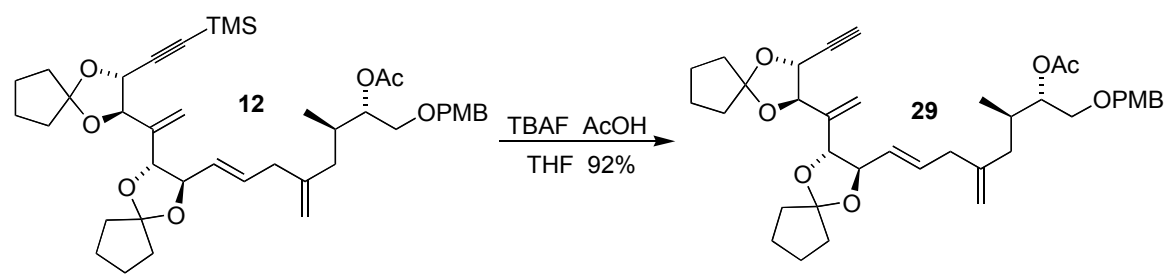

Protected alkyne 12 (940 mg, $1.33 \mathrm{mmol}$ ) was dissolved in $10 \mathrm{~mL}$ of dry THF. Acetic acid $(183 \mathrm{~mL}, 3.2 \mathrm{mmol})$ was then added and the reaction cooled to $0^{\circ} \mathrm{C}$ in an ice bath. TBAF (1M in THF, $1.6 \mathrm{~mL}, 1.6 \mathrm{mmol}$ ) was then added. After approximately 15 min the reaction was 
allowed to warm to room temperature. After $3 \mathrm{~h}$ the reaction appeared done by TLC, and was preabsorbed on silica gel and purified by chromatography (30-35\% $\mathrm{Et}_{2} \mathrm{O} /$ pet. ether) to give 781 mg (92\% yield ) of alkyne 29 as a clear oil: $R_{f}=0.43\left(5 \% \mathrm{Et}_{2} \mathrm{O} / 95 \% \mathrm{CH}_{2} \mathrm{Cl}_{2}\right)$; IR (film from $\left.\mathrm{CH}_{2} \mathrm{Cl}_{2}\right) 3268,2962,2874,2120,1738 \mathrm{~cm}^{-1} ;{ }^{1} \mathrm{H}$ NMR $\left(500 \mathrm{MHz}, \mathrm{CDCl}_{3}\right) \delta 7.23$ (d, J=8.3 Hz, 2H), $6.87(\mathrm{~d}, J=8.5 \mathrm{~Hz}, 2 \mathrm{H}), 5.79-5.85(\mathrm{~m}, 1 \mathrm{H}), 5.46-5.51(\mathrm{~m}, 2 \mathrm{H}), 5.42(\mathrm{~s}, 1 \mathrm{H}), 4.97-5.01(\mathrm{~m}$, $1 \mathrm{H}), 4.80(\mathrm{~s}, 1 \mathrm{H}), 4.75(\mathrm{~s}, 1 \mathrm{H}), 4.39-4.49$ (m, 4H), 4.27 (t, $J=8.1 \mathrm{~Hz}, 1 \mathrm{H}), 4.16$ (d, J=8.3 Hz, $1 \mathrm{H}), 3.81(\mathrm{~s}, 3 \mathrm{H}), 3.52(\mathrm{dd}, J=10.3,6.1 \mathrm{~Hz}, 1 \mathrm{H}), 3.47(\mathrm{dd}, J=10.4,5.1 \mathrm{~Hz}, 1 \mathrm{H}), 2.67-2.77(\mathrm{~m}$, 2H), $2.56(\mathrm{~s}, 1 \mathrm{H}), 2.06(\mathrm{~s}, 3 \mathrm{H}), 2.00-2.05(\mathrm{~m}, 2 \mathrm{H}), 1.79-1.98(\mathrm{~m}, 9 \mathrm{H}), 1.64-1.78(\mathrm{~m}, 8 \mathrm{H}), 0.85(\mathrm{~d}$, $J=6.8 \mathrm{~Hz}, 3 \mathrm{H}) ;{ }^{13} \mathrm{C} \mathrm{NMR}\left(125 \mathrm{MHz}, \mathrm{CDCl}_{3}\right) \delta 170.6,159.2,144.9,141.4,134.1,130.0,129.2$, $128.2,120.4,119.0,116.2,113.8,112.8,82.0,81.2,81.1,80.8,75.2,74.9,72.7,70.5,68.8,55.2$, $39.9,38.5,37.4,37.3,36.7,31.6,30.3,23.8,23.5,23.2,21.1,14.5$.

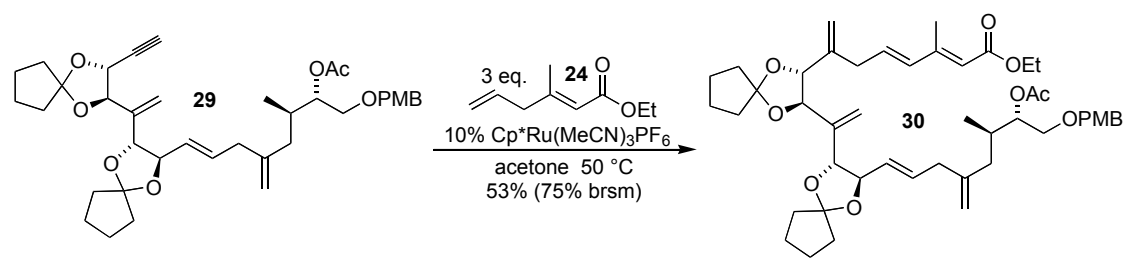

Alkyne 29 (64 mg, $0.101 \mathrm{mmol})$ and alkene 24 (47 mg, $0.307 \mathrm{mmol})$ were dissolved in $300 \mu \mathrm{L}$ of acetone and added to $\mathrm{Cp} * \mathrm{Ru}(\mathrm{MeCN})_{3} \mathrm{PF}_{6}(5.3 \mathrm{mg}, 0.0104 \mathrm{mmol})$. (Note: Acetone was taken from a still over $\mathrm{CaCl}_{2}$ and stirred over $\mathrm{CaCl}_{2}$ for approximately $3 \mathrm{~h}$, and then redisilled). The reaction was immediately submerged in a preheated $50^{\circ} \mathrm{C}$ oil bath. After 90 min the reaction was diluted with $\mathrm{CH}_{2} \mathrm{Cl}_{2}$ and preabsorbed on silica gel. Purification by silica gel chomatography (20-60\% $\mathrm{Et}_{2} \mathrm{O} /$ pet. ether) provided $42 \mathrm{mg}(53 \%)$ of triene product 30 as a $3: 1$ mixture of branched:linear product, $14 \mathrm{mg}(22 \%)$ of recovered alkyne 22 and $30 \mathrm{mg}(63 \%)$ of recovered alkene 24. Data for 30: $R_{f}=0.53\left(5 \% \mathrm{Et}_{2} \mathrm{O} / 95 \% \mathrm{CH}_{2} \mathrm{Cl}_{2}\right)$; IR (film from $\left.\mathrm{CH}_{2} \mathrm{Cl}_{2}\right)$ 2960, 2868, 1736, $1710 \mathrm{~cm}^{-1} ;{ }^{1} \mathrm{H}$ NMR $\left(500 \mathrm{MHz}, \mathrm{CDCl}_{3}\right) \delta 7.22$ (d, $\left.J=8.8 \mathrm{~Hz}, 2 \mathrm{H}\right), 6.86(\mathrm{~d}, J=8.6 \mathrm{~Hz}$, 2H), 6.06-6.15 (m, 2H), 5.78-5.84 (m, 1H), $5.71(\mathrm{bs}, 1 \mathrm{H}), 5.42-5.52(\mathrm{~m}, 3 \mathrm{H}), 5.20$ (bs, 1H), 4.97$5.00(\mathrm{~m}, 2 \mathrm{H}), 4.79$ (bs, 1H), 4.74 (bs, 1H), 4.45 (q, $J=7.5 \mathrm{~Hz}, 2 \mathrm{H}), 4.31$ (d, $J=8.5 \mathrm{~Hz}, 1 \mathrm{H})$, 4.13-4.29 (m, 4H), 4.07 (d, $J=8.3 \mathrm{~Hz}, 1 \mathrm{H}), 3.80$ (s, 3H), 3.44-3.54 (m, 2H), 3.00 (dd, $J=13.9$, $4.9 \mathrm{~Hz}, 0.75 \mathrm{H}), 2.93$ (t, $J=5.6 \mathrm{~Hz}, 0.5 \mathrm{H}), 2.86$ (dd, $J=13.9,5.4 \mathrm{~Hz}, 0.75 \mathrm{H}), 2.65-2.76$ (m, 2H), $2.28(\mathrm{~s}, 3 \mathrm{H}), 2.07-2.10(\mathrm{~m}, 1 \mathrm{H}), 2.06(\mathrm{~s}, 3 \mathrm{H}), 2.00-2.05(\mathrm{~m}, 1 \mathrm{H}), 1.77-1.92(\mathrm{~m}, 9 \mathrm{H}), 1.66-$ $1.74(\mathrm{~m}, 8 \mathrm{H}), 1.28(\mathrm{t}, J=7.1 \mathrm{~Hz}, 3 \mathrm{H}), 0.85(\mathrm{~d}, J=6.6 \mathrm{~Hz}, 3 \mathrm{H}) ;{ }^{13} \mathrm{C} \mathrm{NMR}\left(125 \mathrm{MHz}, \mathrm{CDCl}_{3}\right) \delta$ 
170.6, 167.1, 159.2, 151.9, 145.0, 143.4, 142.2, 142.1, 135.7, 135.0, 133.9, 133.7, 133.4, 130.0, 129.3, 129.2, 128.5, 128.4, 128.2, 119.0, 118.9, 118.8, 118.6, 118.5, 116.4, 115.8, 114.8, 113.7, $112.7,84.4,82.6,82.3,82.2,81.2,80.8,80.4,79.5,75.4,74.8,72.7,68.8,59.7,55.2,39.9,38.5$, 37.4, 37.2, 35.6, 34.4, 31.6, 29.7, 23.5, 23.4, 21.1, 14.3, 14.1, 13.9. Anal calcd for $\mathrm{C}_{47} \mathrm{H}_{64} \mathrm{O}_{10}$ : C, 71.55; H, 8.18. Found: C, 71.44; H, 8.32.
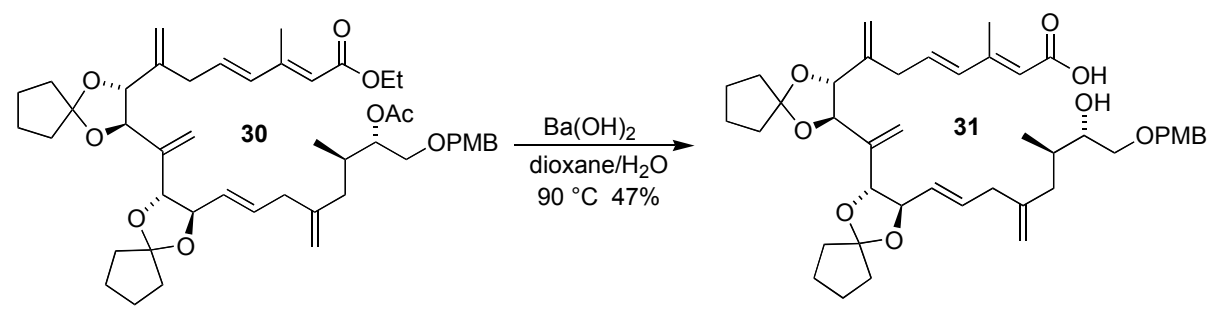

Ester 30 (94 mg, $0.119 \mathrm{mmol})$ was dissolved in $1 \mathrm{~mL}$ of dioxane. Water $(1 \mathrm{~mL})$ was then added followed by barium hydroxide octahydrate $(38 \mathrm{mg}, 0.119 \mathrm{mmol})$. The white suspension was then warmed to $90^{\circ} \mathrm{C}$ (oil bath temperature). After $5 \mathrm{~h}$ the reaction was poured into $25 \mathrm{~mL}$ of $0.5 \mathrm{~N} \mathrm{KHSO}_{4}$ and extracted with $\mathrm{Et}_{2} \mathrm{O}(4 \mathrm{x})$. The extracts were then dried $\left(\mathrm{MgSO}_{4}\right)$ and concentrated. Purification by silica gel chromatography $\left(90 \% \mathrm{Et}_{2} \mathrm{O} /\right.$ pet ether) gave $40 \mathrm{mg}(47 \%)$ of carboxylic acid 31: $R_{f}=0.72\left(100 \% \mathrm{Et}_{2} \mathrm{O}\right)$; IR (neat) $3457,2958,2877,1687 \mathrm{~cm}^{-1} ;{ }^{1} \mathrm{H}$ NMR $\left(500 \mathrm{MHz}, \mathrm{CDCl}_{3}\right) \delta 7.18(\mathrm{~d}, J=7.6 \mathrm{~Hz}, 2 \mathrm{H}), 6.91(\mathrm{~s}, 1 \mathrm{H}), 6.81(\mathrm{~d}, J=8.5 \mathrm{~Hz}, 2 \mathrm{H}), 6.06-6.11$ (m, 2H), $5.75(\mathrm{td}, J=14.9,6.8 \mathrm{~Hz}, 1 \mathrm{H}), 5.67(\mathrm{~s}, 1 \mathrm{H}), 5.35-5.40(\mathrm{~m}, 3 \mathrm{H}), 5.14(\mathrm{~s}, 1 \mathrm{H}), 4.93(\mathrm{~s}$, 1H), 4.71 (s, 2H), 4.41 (abq, $J=15.9,2 \mathrm{H}), 4.24$ (d, $J=8.3 \mathrm{~Hz}, 1 \mathrm{H}), 4.13(\mathrm{t}, J=8.1 \mathrm{~Hz}, 1 \mathrm{H})$, $4.08(\mathrm{~d}, J=8.5 \mathrm{~Hz}, 1 \mathrm{H}), 3.97$ (d, $J=8.1 \mathrm{~Hz}, 1 \mathrm{H}), 3.73(\mathrm{~s}, 3 \mathrm{H}), 3.65$ (td, $J=8.1,3.6,1 \mathrm{H}), 3.40$ (dd, $J=8.6,3.4 \mathrm{~Hz}, 1 \mathrm{H}), 3.33(\mathrm{t}, J=8.4 \mathrm{~Hz}, 1 \mathrm{H}), 2.94$ (dd, $J=14.2,4.6 \mathrm{~Hz}, 1 \mathrm{H}), 2.78$ (dd, $J=$ 13.9, $5.2 \mathrm{~Hz}, 1 \mathrm{H}), 2.60-2.68(\mathrm{~m}, 2 \mathrm{H}), 2.22(\mathrm{~s}, 3 \mathrm{H}), 2.19$ (m, 1H), 2.12 (dd, $J=13.7,5.6 \mathrm{~Hz}, 1 \mathrm{H})$, 1.70-1.83 (m, 8H), 1.58-1.69 (m, 9H), $1.18(\mathrm{~s}, 1 \mathrm{H}), 0.78(\mathrm{~d}, J=6.8 \mathrm{~Hz}, 3 \mathrm{H}) ;{ }^{13} \mathrm{C}$ NMR $(125$ $\left.\mathrm{MHz}, \mathrm{CDCl}_{3}\right) \delta$ 171.5, 159.3, 154.4, 145.5, 143.3, 142.3, 135.6, 134.8, 134.2, 130.0, 129.4, 128.2, 125.5, 119.1, 118.9, 117.6, 116.1, 115.9, 113.9, 112.5, 84.5, 82.3, 81.1, 79.4, 73.0, 72.7, 72.6, 55.3, 40.0, 38.7, 37.4, 37.3, 37.2, 34.5, 34.5, 33.4, 30.3, 23.5, 23.4, 23.4, 14.1, 14.0. 


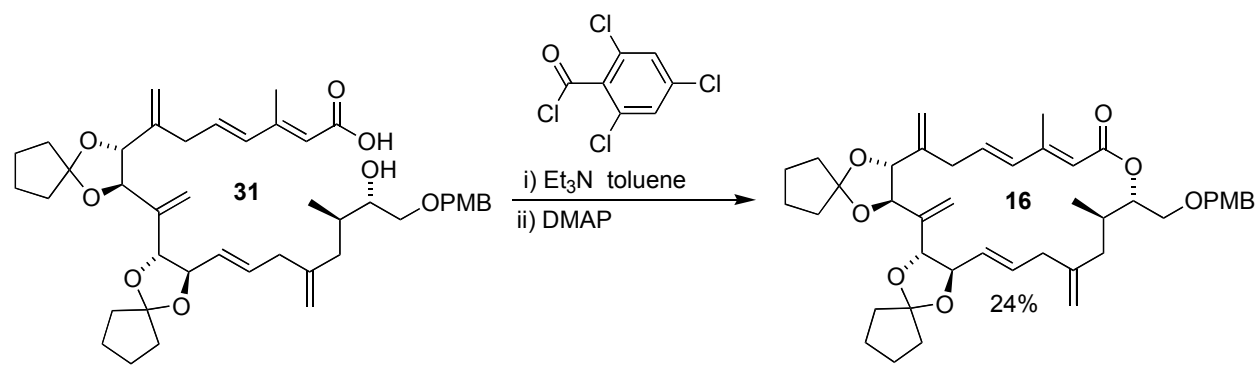

Carboxylic acid 31 (13 mg, $0.018 \mathrm{mmol}$ ) was dissolved in $2 \mathrm{~mL}$ of dry benzene.

Triethylamine $(50 \mu \mathrm{L}, 0.36 \mathrm{mmol})$ was then added followed by 2,4,6-trichlorobenzoyl chloride $(39 \mu \mathrm{L}, 0.25 \mathrm{mmol})$. After approximately $2.5 \mathrm{~h}$ DMAP ( $88 \mathrm{mg}, 0.72 \mathrm{mmol}$ ) was added in $2 \mathrm{~mL}$ of benzene. After $1 \mathrm{~h}$ the reaction was poured into $25 \mathrm{~mL}$ of $0.5 \mathrm{~N} \mathrm{KHSO}_{4}$ and extracted with $\mathrm{Et}_{2} \mathrm{O}(4 \mathrm{x})$. the extracts were then dried $\left(\mathrm{MgSO}_{4}\right)$ and concentrated. Purification by silica gel chomatography (25-40\% $\mathrm{Et}_{2} \mathrm{O}$ /petroleum ether) gave 16 (3 mg, 24\% yield) as a colorless oil: $R_{f}$ $=0.51\left(30 \% \mathrm{Et}_{2} \mathrm{O} / 70 \%\right.$ petroleum ether); IR (neat) $2948,1712 \mathrm{~cm}^{-1} ;{ }^{1} \mathrm{H} \mathrm{NMR}\left(500 \mathrm{MHz}, \mathrm{CDCl}_{3}\right)$ $\delta 7.25(\mathrm{~d}, J=8.5 \mathrm{~Hz}, 2 \mathrm{H}), 6.87(\mathrm{~d}, J=8.1 \mathrm{~Hz}, 2 \mathrm{H}), 6.04-6.19(\mathrm{~m}, 2 \mathrm{H}), 5.69-5.77(\mathrm{~m}, 2 \mathrm{H}), 5.50$ (s, 1H), $5.46(\mathrm{~s}, 1 \mathrm{H}), 5.27(\mathrm{~s}, 1 \mathrm{H}), 5.22(\mathrm{dd}, J=15.9,7.1 \mathrm{~Hz}, 1 \mathrm{H}), 5.15(\mathrm{~s}, 1 \mathrm{H}), 5.04-5.11(\mathrm{~m}$, $1 \mathrm{H}), 4.71(\mathrm{~s}, 1 \mathrm{H}), 4.67(\mathrm{~s}, 1 \mathrm{H}), 4.48(\mathrm{~s}, 2 \mathrm{H}), 4.27(\mathrm{~d}, J=8.0,1 \mathrm{H}), 4.19(\mathrm{~d}, J=8.3 \mathrm{~Hz}, 1 \mathrm{H}), 4.11$ (t, $J=7.6 \mathrm{~Hz}, 1 \mathrm{H}), 3.83(\mathrm{~d}, J=7.8 \mathrm{~Hz}, 1 \mathrm{H}), 3.80(\mathrm{~s}, 3 \mathrm{H}), 3.57-3.64(\mathrm{~m}, 2 \mathrm{H}), 3.13$ (dd, $J=14.4$, $5.9 \mathrm{~Hz}, 1 \mathrm{H}), 3.02(\mathrm{dd}, J=14.4,5.9 \mathrm{~Hz}, 1 \mathrm{H}), 2.52-2.63(\mathrm{~m}, 2 \mathrm{H}), 2.26(\mathrm{~s}, 3 \mathrm{H}), 2.25(\mathrm{~m}, 1 \mathrm{H}), 2.21$ $(\mathrm{dd}, J=13.4,5.9 \mathrm{~Hz}, 1 \mathrm{H}), 1.65-1.91(\mathrm{~m}, 17 \mathrm{H}), 0.94(\mathrm{~d}, J=7.0 \mathrm{~Hz}, 3 \mathrm{H}) ;{ }^{13} \mathrm{C} \mathrm{NMR}(125 \mathrm{MHz}$, $\left.\mathrm{CDCl}_{3}\right) \delta 166.5,159.2,151.5,146.2,143.2,142.6,135.3,133.2,130.1,129.2,128.0,125.5$, 119.0, 118.9, 117.1, 115.2, 113.8, 111.6, 84.6, 82.1, 81.2, 78.4, 75.7, 72.7, 67.4, 55.3, 38.5, 37.6, $37.5,37.3,37.1,35.8,34.2,33.2,30.3,29.7,23.6,23.5,23.4,23.3,16.6,14.3$. MS calcd for $\mathrm{C}_{43} \mathrm{H}_{56} \mathrm{O}_{8}: 700.3975$. Found: 701.3 (APCI+, M+H).
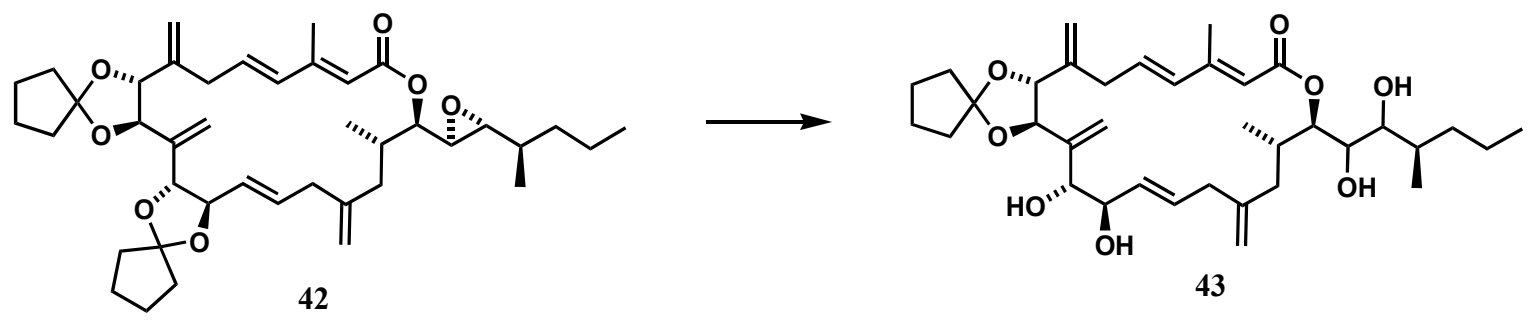

To ketal $42(16.0 \mathrm{mg}, 0.024 \mathrm{mmol})$ was added water $(250 \mu \mathrm{L})$ and acetic acid $(750 \mu \mathrm{L})$. The reaction mixture was heated to $40^{\circ} \mathrm{C}$ for $21 \mathrm{~h}$ and concentrated. Purification by flash column chromatography on silica gel (75\% to $100 \%$ EtOAc in petroleum ether) gave trace amounts of 
several products. Monoketal $43(1.3 \mathrm{mg}, 11 \%)$ was isolated as a single diastereomer and as a colorless oil: $\mathrm{R}_{f}=0.60$ (EtOAc); ${ }^{1} \mathrm{H}$ NMR $\left(500 \mathrm{MHz}, \mathrm{CDCl}_{3}\right) \delta 6.17(\mathrm{dd}, J=15.6,1.2 \mathrm{~Hz}, 1 \mathrm{H})$, 6.10 (ddd, $J=15.4,10.0,4.5 \mathrm{~Hz}, 1 \mathrm{H}), 5.87$ (s, $1 \mathrm{H}), 5.72$ (ddd, $J=15.4,6.2,6.2 \mathrm{~Hz}, 1 \mathrm{H}), 5.51$ (s, 1H), $5.50(\mathrm{~s}, 1 \mathrm{H}), 5.31(\mathrm{~s}, 1 \mathrm{H}), 5.23(\mathrm{ddd}, J=10.9,4.6,1.3 \mathrm{~Hz}, 1 \mathrm{H}), 5.18(\mathrm{~s}, 1 \mathrm{H}), 4.79(\mathrm{~s}, 1 \mathrm{H})$, 4.74 (s, 1H), 4.21 (d, $J=8.6 \mathrm{~Hz}, 1 \mathrm{H}), 4.12(\mathrm{~d}, J=8.5 \mathrm{~Hz}, 1 \mathrm{H}), 4.02$ (t br, $J=5.0 \mathrm{~Hz}, 1 \mathrm{H}), 3.74-$ $3.70(\mathrm{~m}, 3 \mathrm{H}), 3.13(\mathrm{dd}, J=14.3,9.9 \mathrm{~Hz}, 1 \mathrm{H}), 3.05(\mathrm{dd}, J=13.9,3.7 \mathrm{~Hz}, 1 \mathrm{H}), 2.62-2.56(\mathrm{~m}, 2 \mathrm{H})$, $2.33(\mathrm{~d}, J=1.1 \mathrm{~Hz}, 3 \mathrm{H}), 2.31(\mathrm{~m}, 1 \mathrm{H}), 2.15(\mathrm{~d}, J=12.7 \mathrm{~Hz}, 1 \mathrm{H}), 1.92-1.13(\mathrm{~m}, 17 \mathrm{H}), 0.97(\mathrm{~d}, J$ $=6.8 \mathrm{~Hz}, 3 \mathrm{H}), 0.95(\mathrm{~d}, J=6.6 \mathrm{~Hz}, 3 \mathrm{H}), 0.91(\mathrm{t}, J=7.2 \mathrm{~Hz}, 3 \mathrm{H}) ;{ }^{13} \mathrm{C} \mathrm{NMR}\left(101 \mathrm{MHz}, \mathrm{CDCl}_{3}\right) \delta$ 166.0, 153.6, 145.2, 145.0, 142.3, 137.0, 135.1, 131.4, 129.2, 118.9, 118.3, 117.7, 115.3, 113.2, 85.4, 79.6, 77.2, 75.7, 74.9, 74.1, 70.5, 40.2, 39.3, 37.5, 37.3, 35.4, 35.3, 34.1, 33.7, 23.4, 23.4, 19.8, 15.8, 15.6, 14.3, 14.1; IR (neat) 3411 (br), 2949, 1709, 1610, $1148 \mathrm{~cm}^{-1}$; ESIMS calcd for $\mathrm{C}_{36} \mathrm{H}_{54} \mathrm{O}_{8} \mathrm{Na}\left(\mathrm{M}^{+}+\mathrm{Na}\right)$ 637.4, found 637.5.
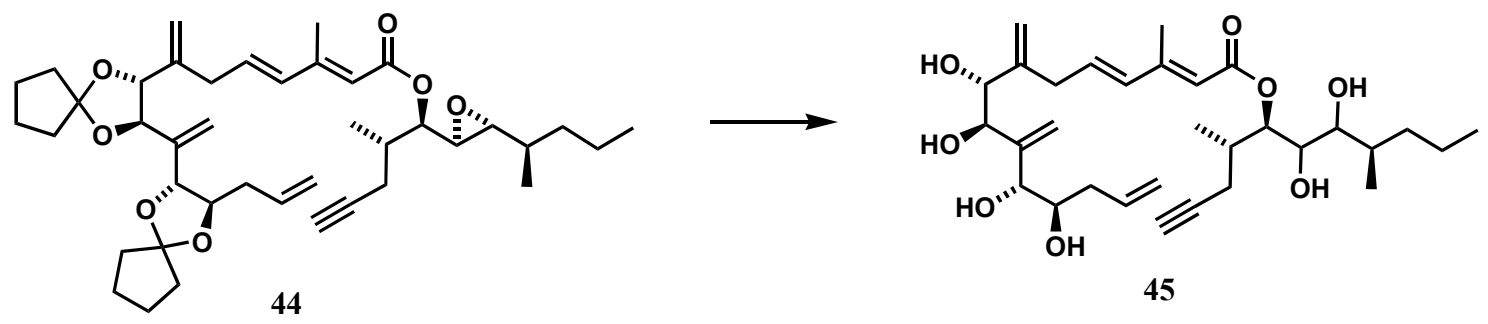

To ketal $44(17 \mathrm{mg}, 0.026 \mathrm{mmol})$ was added acetic acid $(750 \mu \mathrm{L})$ and water $(250 \mu \mathrm{L})$.

The reaction mixture was heated to $40{ }^{\circ} \mathrm{C}$ for $19 \mathrm{~h}$ and concentrated. Purification by flash column chromatography on silica gel (50\% to $75 \%$ to $100 \%$ EtOAc in petroleum ether) followed again by flash column chromatography on silica gel $\left(5 \%\right.$ to $10 \% \mathrm{MeOH}$ in $\left.\mathrm{CH}_{2} \mathrm{Cl}_{2}\right)$ gave trace amounts of several products. Notably, $\mathbf{4 5}(2.3 \mathrm{mg}, 17 \%)$ was isolated as a single diastereomer and as an amorphous solid: $\mathrm{R}_{f}=0.34\left(10 \% \mathrm{MeOH}\right.$ in $\left.\mathrm{CH}_{2} \mathrm{Cl}_{2}\right) ;{ }^{1} \mathrm{H} \mathrm{NMR}\left(500 \mathrm{MHz}, \mathrm{CDCl}_{3}\right) \delta$ 6.19-6.16 $(\mathrm{m}, 2 \mathrm{H}), 5.86(\mathrm{~m}, 1 \mathrm{H}), 5.83(\mathrm{~s}, 1 \mathrm{H}), 5.30-5.26(\mathrm{~m}, 3 \mathrm{H}), 5.17(\mathrm{~m}, 1 \mathrm{H}), 5.16(\mathrm{~s}, 1 \mathrm{H}), 5.05(\mathrm{~s}, 1 \mathrm{H})$, 4.27 (s br, 2H), 4.10 (d, $J=4.5 \mathrm{~Hz}, 1 \mathrm{H}), 4.04$ (d br, $J=5.4 \mathrm{~Hz}, 1 \mathrm{H}$ ), 3.84 (ddd, $J=7.9,4.3,4.3$ Hz, 1H), 3.67 (m, 1H), 3.04 (dd, $J=15.9,3.5 \mathrm{~Hz}, 1 \mathrm{H}$ ), 2.92 (d br, $J=16.5 \mathrm{~Hz}, 1 \mathrm{H}$ ), 2.39 (ddd, $J$ $=14.4,4.9,4.9 \mathrm{~Hz}, 1 \mathrm{H}), 2.30$ (s, 3H), $2.25(\mathrm{ddd}, J=5.9,5.9,2.4 \mathrm{~Hz}, 1 \mathrm{H}), 1.98(\mathrm{dd}, J=2.7,2.7$ $\mathrm{Hz}, 1 \mathrm{H}), 1.82(\mathrm{~m}, 1 \mathrm{H}), 1.69(\mathrm{~m}, 1 \mathrm{H}), 1.46-1.20(\mathrm{~m}, 7 \mathrm{H}), 1.13(\mathrm{~d}, J=6.7 \mathrm{~Hz}, 3 \mathrm{H}), 0.99(\mathrm{~d}, J=6.7$ $\mathrm{Hz}, 3 \mathrm{H}), 0.90(\mathrm{t}, J=7.2 \mathrm{~Hz}, 3 \mathrm{H}) ;{ }^{13} \mathrm{C} \mathrm{NMR}\left(101 \mathrm{MHz}, \mathrm{CDCl}_{3}\right) \delta 166.1,153.8,147.7,146.0$, 135.5, 134.5, 134.1, 118.6, 117.7, 116.6, 114.2, 81.8, 77.8, 77.2, 76.3, 75.2, 73.00, 72.97, 71.4, 
70.4, 37.9, 36.5, 35.5, 34.6, 34.3, 22.3, 19.8, 15.6, 15.5, 14.3, 14.1; IR (neat) 3303 (br), 2922, $1709,1610,1153 \mathrm{~cm}^{-1}$; ESIMS calcd for $\mathrm{C}_{31} \mathrm{H}_{48} \mathrm{O}_{8} \mathrm{Na}\left(\mathrm{M}^{+}+\mathrm{Na}\right)$ 571.3, found 571.3.
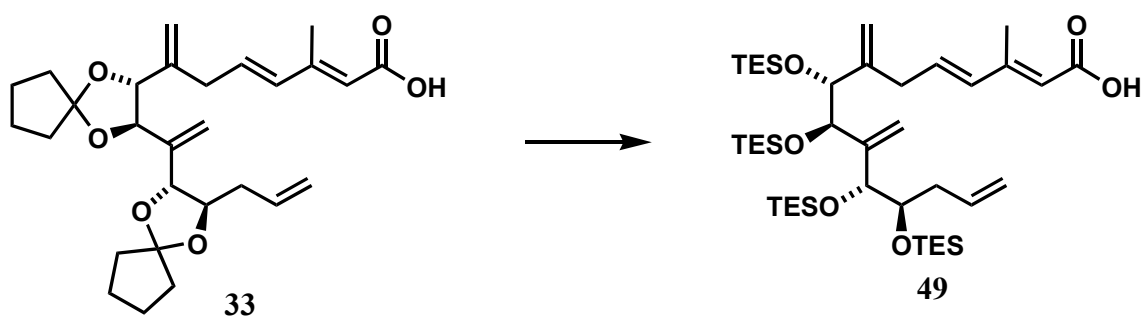

To ketal $33(117 \mathrm{mg}, 0.25 \mathrm{mmol})$ at room temperature was added acetic acid $(1.5 \mathrm{~mL})$ and water $(500 \mu \mathrm{L})$. The reaction mixture was heated to $40{ }^{\circ} \mathrm{C}$ for $21 \mathrm{~h}$ and concentrated to give tetraol $\mathbf{4 8}$ which was used in the next step without further purification. To a solution of tetraol 48, prepared in the previous step, in THF $(5 \mathrm{~mL})$ at $0{ }^{\circ} \mathrm{C}$ was added $i-\operatorname{Pr}_{2} \mathrm{NEt}(350 \mu \mathrm{L}, 260 \mathrm{mg}$, $2.0 \mathrm{mmol})$ and TESOTf $(340 \mu \mathrm{L}, 398 \mathrm{mg}, 1.50 \mathrm{mmol})$. The reaction mixture was stirred at $0{ }^{\circ} \mathrm{C}$ for $30 \mathrm{~min}$, quenched with $1 \mathrm{M} \mathrm{HCl}(5 \mathrm{~mL})$, stirred for $5 \mathrm{~min}$, and diluted with ether and water. The aqueous phase was extracted with ether $(3 \mathrm{x})$ and the combined organic extracts were washed with brine $(1 \mathrm{x})$, dried over $\mathrm{MgSO}_{4}$, and concentrated. Purification by flash column chromatography on silica gel (5\% to $10 \%$ to $15 \%$ to $20 \%$ EtOAc in petroleum ether) followed again by flash column chromatography on silica gel $(0.5 \%$ to $1 \%$ to $1.5 \%$ to $2 \% \mathrm{MeOH}$ in $\mathrm{CH}_{2} \mathrm{Cl}_{2}$ ) a gave silyl ether 49 (148 mg, $\left.75 \%\right)$ as a colorless oil: $\mathrm{R}_{f}=0.45(30 \%$ EtOAc in petroleum ether); ${ }^{1} \mathrm{H}$ NMR (400 MHz, $\mathrm{CDCl}_{3}$ ) $\delta$ 6.22-6.11 (m, $2 \mathrm{H}$ ), 5.83 (dddd, $J=17.1,10.2$, 8.1, $7.8 \mathrm{~Hz}, 1 \mathrm{H}), 5.74(\mathrm{~s}, 1 \mathrm{H}), 5.32$ (s, 2H), 5.04-4.98 (m, 3H), 4.84 (s, 1H), 4.49 (d, J=4.0 Hz, $1 \mathrm{H}), 4.35$ (d, $J=3.4 \mathrm{~Hz}, 1 \mathrm{H}), 4.15$ (d, $J=4.1 \mathrm{~Hz}, 1 \mathrm{H}), 3.74$ (ddd, $J=7.9,3.8,3.8 \mathrm{~Hz}, 1 \mathrm{H}), 3.01$ (dd, $J=16.0,4.9 \mathrm{~Hz}, 1 \mathrm{H}), 2.94$ (dd, $J=16.9,6.9 \mathrm{~Hz}, 1 \mathrm{H}), 2.38(\mathrm{dm}, J=14.3 \mathrm{~Hz}, 1 \mathrm{H}), 2.31$ (s, $3 \mathrm{H}), 2.04$ (ddd, $J=14.3,8.2,8.2 \mathrm{~Hz}, 1 \mathrm{H}), 0.99-0.93(\mathrm{~m}, 36 \mathrm{H}), 0.67-0.56(\mathrm{~m}, 24 \mathrm{H}) ;{ }^{13} \mathrm{C}$ NMR $\left(101 \mathrm{MHz}, \mathrm{CDCl}_{3}\right) \delta$ 172.7, 155.1, 148.4, 146.9, 137.1, 136.3, 135.0, 117.1, 116.2, 113.6, 113.5, 80.1, 76.7, 74.8, 74.6, 36.8, 36.3, 14.0, 7.15, 7.07, 7.03, 7.01, 5.5, 5.2, 4.9 (two carbons); IR (neat) 3067 (br), 2959, 1682, 1610, $1085 \mathrm{~cm}^{-1}$; ESIMS calcd for $\mathrm{C}_{42} \mathrm{H}_{83} \mathrm{O}_{6} \mathrm{Si}_{4}\left(\mathrm{M}^{+}+\mathrm{H}\right) 795.5$, found 795.5; optical rotation $[\alpha]_{\mathrm{D}}^{23}+32^{\circ}\left(c\right.$ 1.0, $\left.\mathrm{CH}_{2} \mathrm{Cl}_{2}\right)$; Anal. Calcd for $\mathrm{C}_{42} \mathrm{H}_{82} \mathrm{O}_{6} \mathrm{Si}_{4}$ : C, 63.42; H, 10.39. Found: C, 63.25; H, 10.25 . 

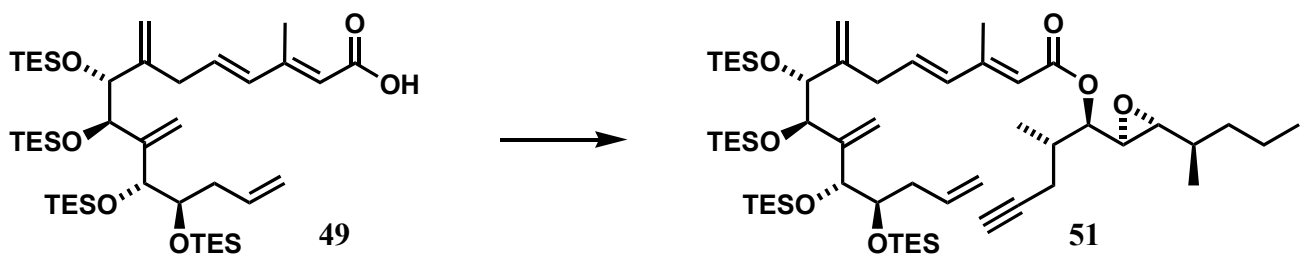

To $\left[\mathrm{RuCl}_{2}(p \text {-cymene })\right]_{2}(11 \mathrm{mg}, 0.018 \mathrm{mmol})$ was added a solution of acid 49 (142 mg, $0.18 \mathrm{mmol})$ in $\mathrm{PhMe}(2.9 \mathrm{~mL})$ at room temperature. Ethoxyacetylene (47 $\mu \mathrm{L}, 50 \% \mathrm{wt}$. in hexanes, $0.27 \mathrm{mmol}$ ) was added and the reaction mixture was stirred at room temperature for $2 \mathrm{~h}$ and concentrated under a stream of argon. A solution of alcohol $\mathbf{5 0}(113 \mathrm{mg}, 0.537 \mathrm{mmol})$ in DCE $(3.2 \mathrm{~mL})$ was added via cannula followed by CSA $(50 \mu \mathrm{L}, 0.14 \mathrm{M}$ in DCE, $7 \mu \mathrm{mol})$. The reaction mixture was stirred at room temperature for $2 \mathrm{~h}$, filtered through silica gel, and concentrated. Purification by flash column chromatography on silica gel ( $2 \%$ to $5 \%$ to $15 \%$ to 100\% EtOAc in petroleum ether) gave ester 51 (64 mg, 36\%) as a colorless oil and recovered acid 49 (30 mg, 21\%). Data for 51: $\mathrm{R}_{f}=0.33$ (5\% EtOAc in petroleum ether); ${ }^{1} \mathrm{H}$ NMR (500 $\left.\mathrm{MHz}, \mathrm{CDCl}_{3}\right) \delta$ 6.18-6.09 (m, 2H), 5.82 (dddd, $\left.J=17.0,10.1,7.8,6.7 \mathrm{~Hz}, 1 \mathrm{H}\right), 5.70(\mathrm{~s}, 1 \mathrm{H})$, $5.31(\mathrm{~s}, 2 \mathrm{H}), 5.03-4.98(\mathrm{~m}, 3 \mathrm{H}), 4.83(\mathrm{~s}, 1 \mathrm{H}), 4.70(\mathrm{dd}, J=6.6,4.3 \mathrm{~Hz}, 1 \mathrm{H}), 4.48(\mathrm{~d}, J=4.2 \mathrm{~Hz}$, $1 \mathrm{H}), 4.34(\mathrm{~d}, J=3.4 \mathrm{~Hz}, 1 \mathrm{H}), 4.14(\mathrm{~d}, J=4.0 \mathrm{~Hz}, 1 \mathrm{H}), 3.70(\mathrm{ddd}, J=8.3,3.7,3.7 \mathrm{~Hz}, 1 \mathrm{H}), 3.00$ $(\mathrm{dd}, J=16.0,5.0 \mathrm{~Hz}, 1 \mathrm{H}), 2.92(\mathrm{dd}, J=15.9,6.5 \mathrm{~Hz}, 1 \mathrm{H}), 2.81(\mathrm{dd}, J=7.4,2.1 \mathrm{~Hz}, 1 \mathrm{H}), 2.75$ (dd, $J=6.6,2.1 \mathrm{~Hz}, 1 \mathrm{H}), 2.40-2.31(\mathrm{~m}, 2 \mathrm{H}), 2.29$ (d, $J=1.0 \mathrm{~Hz}, 3 \mathrm{H}), 2.19$ (dd, $J=7.9,2.6 \mathrm{~Hz}$, 1H), 2.14 (m 1H), 2.03 (ddd, $J=14.3,8.2,8.2 \mathrm{~Hz}, 1 \mathrm{H}), 1.98(\mathrm{dd}, J=2.3,2.3 \mathrm{~Hz}, 1 \mathrm{H}), 1.49-1.22$ $(\mathrm{m}, 5 \mathrm{H}), 1.14(\mathrm{~d}, J=6.6 \mathrm{~Hz}, 3 \mathrm{H}), 0.98-0.92(\mathrm{~m}, 39 \mathrm{H}), 0.88(\mathrm{t}, J=6.8 \mathrm{~Hz}, 3 \mathrm{H}), 0.66-0.56(\mathrm{~m}$, $24 \mathrm{H}) ;{ }^{13} \mathrm{C}$ NMR $\left(126 \mathrm{MHz}, \mathrm{CDCl}_{3}\right) \delta$ 166.1, 153.5, 148.4, 146.9, 137.1, 135.7, 135.0, 117.0, $116.2,113.6,113.4,82.2,80.1,76.1,74.8,74.6,74.1,69.7,62.5,55.6,36.8,36.7,36.6,35.3$, 35.1, 22.4, 20.0, 15.6, 14.3, 14.2, 13.9, 7.2, 7.1, 7.03, 7.02, 5.4, 5.2, 4.9 (two carbons); IR (neat) $3315,2957,1719,1611,1007 \mathrm{~cm}^{-1}$; ESIMS calcd for $\mathrm{C}_{55} \mathrm{H}_{102} \mathrm{O}_{7} \mathrm{Si}_{4} \mathrm{Na}\left(\mathrm{M}^{+}+\mathrm{Na}\right) 1009.7$, found 1009.5; optical rotation $[\alpha]_{\mathrm{D}}^{24}+41^{\circ}\left(c 0.50, \mathrm{CH}_{2} \mathrm{Cl}_{2}\right)$.
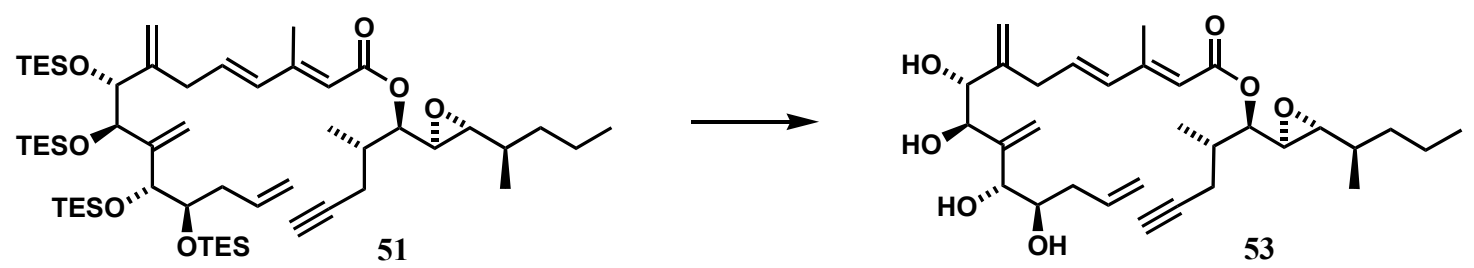
To a solution of silyl ether $51(73 \mathrm{mg}, 0.074 \mathrm{mmol})$ in THF $(1.7 \mathrm{~mL})$ at room temperature was added TBAF (438 $\mu \mathrm{L}, 1 \mathrm{M}$ in THF, $0.44 \mathrm{mmol})$ and acetic acid $(25 \mu \mathrm{L}, 26 \mathrm{mg}, 0.44 \mathrm{mmol})$. The reaction mixture was stirred at room temperature for $25 \mathrm{~h}$ and concentrated. Purification by flash column chromatography on silica gel (5\% to $7.5 \%$ to $10 \% \mathrm{MeOH}$ in $\left.\mathrm{CH}_{2} \mathrm{Cl}_{2}\right)$ gave tetraol $53(21 \mathrm{mg}, 54 \%)$ as an amorphous solid: $\mathrm{R}_{f}=0.45\left(10 \% \mathrm{MeOH}\right.$ in $\left.\mathrm{CH}_{2} \mathrm{Cl}_{2}\right) ;{ }^{1} \mathrm{H} \mathrm{NMR}(500 \mathrm{MHz}$, $\left.\mathrm{CDCl}_{3}\right) \delta$ 6.21-6.14 (m, 2H), 5.85 (dddd, $\left.J=16.9,10.6,7.6,6.8 \mathrm{~Hz}, 1 \mathrm{H}\right), 5.72(\mathrm{~s}, 1 \mathrm{H}), 5.31-5.24$ $(\mathrm{m}, 2 \mathrm{H}), 5.27(\mathrm{~s}, 1 \mathrm{H}), 5.17(\mathrm{~m}, 1 \mathrm{H}), 5.15(\mathrm{~s}, 1 \mathrm{H}), 5.04(\mathrm{~s}, 1 \mathrm{H}), 4.71(\mathrm{dd}, J=6.5,4.3 \mathrm{~Hz}, 1 \mathrm{H})$, $4.26(\mathrm{~s}, 2 \mathrm{H}), 4.10(\mathrm{~d}, J=4.5 \mathrm{~Hz}, 1 \mathrm{H}), 3.84(\mathrm{ddd}, J=8.2,4.8,4.8 \mathrm{~Hz}, 1 \mathrm{H}), 3.03$ (dd, $J=17.2,5.5$ $\mathrm{Hz}, 1 \mathrm{H}), 2.91(\mathrm{dd}, J=17.0,6.2 \mathrm{~Hz}, 1 \mathrm{H}), 2.81(\mathrm{dd}, J=7.4,2.1 \mathrm{~Hz}, 1 \mathrm{H}), 2.75(\mathrm{dd}, J=6.5,2.1 \mathrm{~Hz}$, $1 \mathrm{H}), 2.42-2.34(\mathrm{~m}, 2 \mathrm{H}), 2.32(\mathrm{dd}, J=4.6,2.7 \mathrm{~Hz}, 1 \mathrm{H}), 2.27(\mathrm{~d}, J=1.1 \mathrm{~Hz}, 3 \mathrm{H}), 2.19(\mathrm{dd}, J=$ $7.8,2.6 \mathrm{~Hz}, 1 \mathrm{H}), 2.13(\mathrm{~m}, 1 \mathrm{H}), 1.98(\mathrm{dd}, J=2.7,2.7 \mathrm{~Hz}, 1 \mathrm{H}), 1.49-1.23(\mathrm{~m}, 5 \mathrm{H}), 1.13(\mathrm{~d}, J=6.6$ $\mathrm{Hz}, 3 \mathrm{H}), 0.882(\mathrm{t}, J=7.0 \mathrm{~Hz}, 3 \mathrm{H}), 0.876(\mathrm{~d}, J=6.2 \mathrm{~Hz}, 3 \mathrm{H}) ;{ }^{13} \mathrm{C} \mathrm{NMR}\left(126 \mathrm{MHz}, \mathrm{CDCl}_{3}\right) \delta$ $165.9,152.9,147.7,146.1,135.6,134.2,134.0,118.6,117.9,116.6,114.1,82.2,76.5,76.4,75.2$, $74.1,72.9,69.8,62.5,55.6,38.0,36.6,36.3,35.3,35.0,22.5,20.0,15.7,14.3,14.2$, 14.0; IR (neat) 3294 (br), 2913, 1719, 1610, $1143 \mathrm{~cm}^{-1}$; ESIMS calcd for $\mathrm{C}_{31} \mathrm{H}_{46} \mathrm{O}_{7} \mathrm{Na}\left(\mathrm{M}^{+}+\mathrm{Na}\right) 553.3$, found 553.3; optical rotation $[\alpha]_{\mathrm{D}}{ }^{26}+53^{\circ}\left(c 0.10, \mathrm{CH}_{2} \mathrm{Cl}_{2}\right)$.
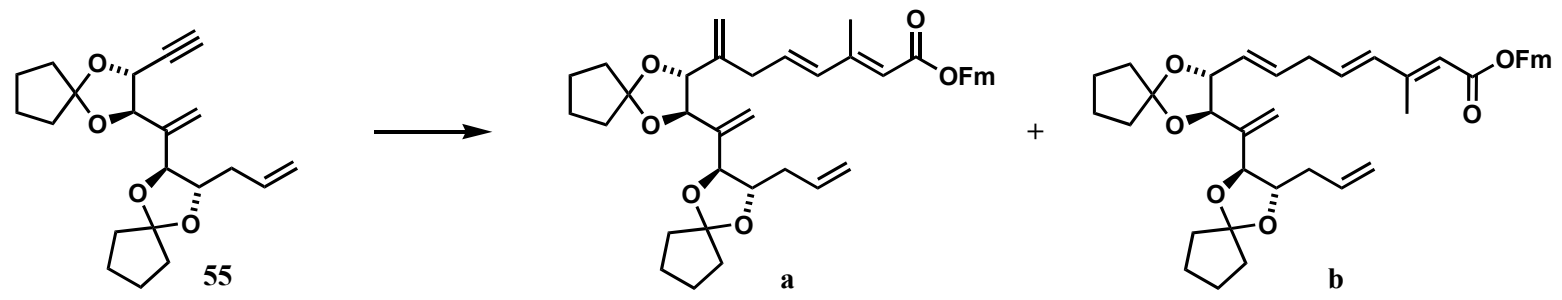

A solution of alkyne $55(576 \mathrm{mg}, 1.67 \mathrm{mmol})$ and alkene $5(2.581 \mathrm{~g}, 8.48 \mathrm{mmol})$ in DCE $(4.3 \mathrm{~mL})$ was degassed by bubbling argon through the solution for $15 \mathrm{~min}$, the reaction mixture was heated to $50^{\circ} \mathrm{C}$, and $\mathrm{Cp} * \mathrm{Ru}(\mathrm{MeCN})_{3} \mathrm{PF}_{6}(87 \mathrm{mg}, 0.17 \mathrm{mmol})$ was added in one portion. After $1 \mathrm{~h}$, the reaction mixture was filtered through silica gel and concentrated. Purification by flash column chromatography on silica gel (3\% to 6\% to 9\% EtOAc in petroleum ether) gave branched alkene a (251 mg, 23\%), linear alkene b (94 mg, 9\%) and recovered alkyne 55 (233 $\mathrm{mg}, 40 \%)$ as colorless oils. Data for $\mathbf{a}: \mathrm{R}_{f}=0.30$ (10\% EtOAc in petroleum ether); ${ }^{1} \mathrm{H}$ NMR $(500$ $\left.\mathrm{MHz}, \mathrm{CDCl}_{3}\right) \delta 7.77$ (d, J=7.6 Hz, 2H), $7.62(\mathrm{~d}, J=7.5 \mathrm{~Hz}, 2 \mathrm{H}), 7.41$ (dd, $\left.J=7.5,7.5 \mathrm{~Hz}, 2 \mathrm{H}\right)$, $7.32(\mathrm{dd}, J=7.4,7.4 \mathrm{~Hz}, 2 \mathrm{H}), 6.22-6.14$ (m, 2H), 5.89 (dddd, $J=17.1,13.7,10.1,6.7 \mathrm{~Hz}, 1 \mathrm{H})$, $5.83(\mathrm{~s}, 1 \mathrm{H}), 5.43(\mathrm{~s}, 1 \mathrm{H}), 5.38(\mathrm{~s}, 1 \mathrm{H}), 5.25(\mathrm{~s}, 1 \mathrm{H}), 5.16-5.10(\mathrm{~m}, 2 \mathrm{H}), 5.00(\mathrm{~s}, 1 \mathrm{H}), 4.42(\mathrm{~d}, J=$ S13 
$7.2 \mathrm{~Hz}, 2 \mathrm{H}), 4.32-4.24(\mathrm{~m}, 3 \mathrm{H}), 4.18$ (d, $J=7.8 \mathrm{~Hz}, 1 \mathrm{H}), 3.93$ (ddd, $J=7.7,7.7,3.8 \mathrm{~Hz}, 1 \mathrm{H})$, $3.03(\mathrm{dd}, J=16.6,5.9 \mathrm{~Hz}, 1 \mathrm{H}), 2.92(\mathrm{dd}, J=16.6,6.7 \mathrm{~Hz}, 1 \mathrm{H}), 2.52(\mathrm{~m}, 1 \mathrm{H}), 2.31$ (ddd, $J=$ 14.5, 7.5, 7.5 Hz, 1H), 2.27 (s, 3H), 1.93-1.62 (m, 16H); $\left.{ }^{13} \mathrm{C} \mathrm{NMR} \mathrm{(126} \mathrm{MHz,} \mathrm{CDCl}_{3}\right) \delta$ 167.0, 153.0, 144.0, 143.3, 142.8, 141.3, 135.5, 134.4, 134.3, 127.7, 127.0, 125.1, 119.9, 119.0, 118.8, $117.8,117.6,117.2,114.7,83.0,80.3,80.2,79.4,65.7,46.8,37.6,37.4,37.30,37.25,36.3,35.1$, 23.5, 23.4 (2 carbons), 23.3, 13.9; IR (neat) 2959, 1715, 1613, 1147, $1107 \mathrm{~cm}^{-1}$; EIMS $\mathrm{m} / z 180$ (21), 165 (20); HREIMS calcd for $\mathrm{C}_{42} \mathrm{H}_{48} \mathrm{O}_{6} 648.3451$, found 648.3456; optical rotation $[\alpha]^{24}{ }_{D}$ $+21^{\circ}\left(c 0.50, \mathrm{CH}_{2} \mathrm{Cl}_{2}\right)$. Data for $\mathbf{b}: \mathrm{R}_{f}=0.26\left(10 \%\right.$ EtOAc in petroleum ether); ${ }^{1} \mathrm{H}$ NMR (500 $\left.\mathrm{MHz}, \mathrm{CDCl}_{3}\right) \delta 7.77$ (d, $\left.J=7.5 \mathrm{~Hz}, 2 \mathrm{H}\right), 7.62(\mathrm{~d}, J=7.5 \mathrm{~Hz}, 2 \mathrm{H}), 7.41$ (t, $\left.J=7.5 \mathrm{~Hz}, 2 \mathrm{H}\right), 7.32$ (t, $J=7.5 \mathrm{~Hz}, 2 \mathrm{H}), 6.19-6.13(\mathrm{~m}, 2 \mathrm{H}), 5.93-5.80$ (m, 2H), 5.83 (s, 1H), 5.56 (dd, $J=15.4,6.8$ $\mathrm{Hz}, 1 \mathrm{H}), 5.48-5.09(\mathrm{~m}, 3 \mathrm{H}), 5.38(\mathrm{~s}, 1 \mathrm{H}), 4.42(\mathrm{~d}, J=7.2 \mathrm{~Hz}, 1 \mathrm{H}), 4.30-4.06(\mathrm{~m}, 5 \mathrm{H}), 3.91(\mathrm{~m}$, 1H), $2.95(\mathrm{dd}, J=5.9,5.9 \mathrm{~Hz}, 1 \mathrm{H}), 2.52(\mathrm{dm}, J=10.4 \mathrm{~Hz}, 1 \mathrm{H}), 2.33-2.27(\mathrm{~m}, 2 \mathrm{H}), 2.25(\mathrm{~s}, 3 \mathrm{H})$, $1.89-1.65(\mathrm{~m}, 16 \mathrm{H}) ;{ }^{13} \mathrm{C} \mathrm{NMR}\left(126 \mathrm{MHz}, \mathrm{CDCl}_{3}\right) \delta 166.9,152.9,144.0,142.7,141.2,134.7$, 134.3, 134.2, 132.3, 128.2, 127.7, 127.0, 125.1, 119.9, 118.9, 118.8, 117.9, 117.2, 116.5, 81.9, $80.9,80.01,79.96,65.7,46.8,37.6,37.5,37.3,37.2,36.4,35.5,23.50,23.47,23.4,23.3,13.8$.
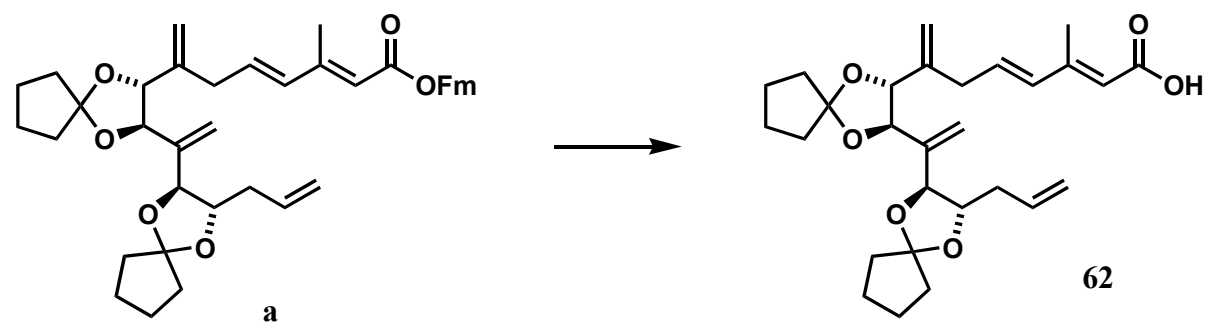

To a solution of ester a ( $310 \mathrm{mg}, 0.48 \mathrm{mmol})$ in $\mathrm{CH}_{2} \mathrm{Cl}_{2}(14 \mathrm{~mL})$ at room temperature was added piperidine $(1.6 \mathrm{~mL}, 1.4 \mathrm{~g}, 16 \mathrm{mmol})$. After $3 \mathrm{~h}$ at room temperature, the reaction mixture was diluted with ether, water, and $1 \mathrm{M} \mathrm{HCl}$. The aqueous phase was extracted with ether $(3 \mathrm{x})$ and the combined organic extracts were washed with brine $(1 \mathrm{x})$, dried over $\mathrm{MgSO}_{4}$, and concentrated. Purification by flash column chromatography on silica gel (30\% to $50 \%$ EtOAc in petroleum ether) gave acid $62(198 \mathrm{mg}, 88 \%)$ as a colorless oil: $\mathrm{R}_{f}=0.41(40 \%$ EtOAc in petroleum ether); ${ }^{1} \mathrm{H}$ NMR (500 MHz, $\mathrm{CDCl}_{3}$ ) $\delta$ 6.18-6.14 (m, 2H), 5.88 (dddd, $\mathrm{J}=17.1,13.7$, 10.3, $6.7 \mathrm{~Hz}, 1 \mathrm{H}), 5.74(\mathrm{~d}, \mathrm{~J}=0.9 \mathrm{~Hz}, 1 \mathrm{H}), 5.41(\mathrm{~s}, 1 \mathrm{H}), 5.36(\mathrm{~s}, 1 \mathrm{H}), 5.23(\mathrm{~s}, 1 \mathrm{H}), 5.15-5.08(\mathrm{~m}$, 2H), $4.97(\mathrm{~d}, \mathrm{~J}=1.1 \mathrm{~Hz}, 1 \mathrm{H}), 4.29(\mathrm{~d}, \mathrm{~J}=8.4 \mathrm{~Hz}, 1 \mathrm{H}), 4.26(\mathrm{~d}, \mathrm{~J}=8.4 \mathrm{~Hz}, 1 \mathrm{H}), 4.16(\mathrm{~d}, \mathrm{~J}=7.7$ Hz, 1H), 3.91 (ddd, J = 7.7, 7.7, 3.8 Hz, 1H), 3.02 (dd, J = 16.2, 4.5 Hz, 1H), 2.91 (dd, J = 16.1, 
$5.3 \mathrm{~Hz}, 1 \mathrm{H}), 2.51(\mathrm{dm}, \mathrm{J}=14.7 \mathrm{~Hz}, 1 \mathrm{H}), 2.31(\mathrm{~m}, 1 \mathrm{H}), 2.29(\mathrm{~d}, \mathrm{~J}=1.0 \mathrm{~Hz}, 3 \mathrm{H}), 1.90-1.62(\mathrm{~m}$, $17 \mathrm{H}) ;{ }^{13} \mathrm{C}$ NMR $\left(126 \mathrm{MHz}, \mathrm{CDCl}_{3}\right) \delta 172.0,154.7,143.2,142.8,135.5,135.1,134.3,119.0$, 118.8, 117.6, 117.4, 117.3, 114.8, 83.1, 80.3, 80.2, 79.4, 37.6, 37.4, 37.30, 37.25, 36.3, 35.1, 23.5, 23.41, 23.40, 23.3, 14.1; IR (neat) 3076 (br), 2959, 1682, 1610, $1107 \mathrm{~cm}^{-1}$; MALDIMS calcd for $\mathrm{C}_{28} \mathrm{H}_{38} \mathrm{O}_{6} \mathrm{Na}\left(\mathrm{M}^{+}+\mathrm{Na}\right) 493.3$, found 493.2; optical rotation $[\alpha]_{\mathrm{D}}{ }^{28}+28^{\circ}$ (c 0.50, $\mathrm{CH}_{2} \mathrm{Cl}_{2}$ ). 
${ }^{1} \mathrm{H} \mathrm{NMR}$ data for $\mathbf{1}$ in $\mathrm{CDCl}_{3}$.

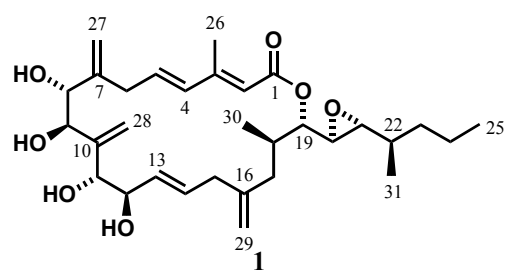

\begin{tabular}{|c|c|c|c|c|c|c|}
\hline & $\underline{\text { Isomer } 1}$ & & & $\underline{\text { Natural Product }}$ & & \\
\hline Proton \# & $\delta_{\mathrm{H}}$ & Mult. & $J$ & $\delta_{\mathrm{H}}$ & Mult. & $J$ \\
\hline 2 & 5.80 & $\mathrm{~s}$ & - & 5.80 & $\mathrm{~s}$ & - \\
\hline 4 & 6.20 & d & 15.5 & 6.27 & $\mathrm{~d}$ & 15.6 \\
\hline 5 & 6.09 & ddd & $15.5,10.3,4.5$ & 6.09 & ddd & $15.6,8.8,4.8$ \\
\hline $6 a$ & 3.22 & $\mathrm{dd}$ & $14.9,4.5$ & 3.20 & $\mathrm{dd}$ & $14.6,4.8$ \\
\hline $6 \mathrm{~b}$ & 3.12 & $\mathrm{dd}$ & $14.9,10.3$ & 3.13 & $\mathrm{dd}$ & $14.6,8.8$ \\
\hline 8 & 4.23 & $\mathrm{~s} b r$ & - & 4.42 & $\mathrm{~s} b r$ & - \\
\hline 9 & 4.29 & $\mathrm{~s} b r$ & - & 4.58 & $\mathrm{~s} b r$ & - \\
\hline 11 & 3.84 & $\mathrm{sbr}$ & - & 4.09 & $\mathrm{~s} b r$ & - \\
\hline 12 & 4.21 & $\mathrm{~s}$ br & - & 4.22 & $\mathrm{~s} b r$ & - \\
\hline 13 & 5.28 & dd & $15.3,2.6$ & 5.51 & $\mathrm{dd}$ & $15.4,4.1$ \\
\hline 14 & $5.78-5.73$ & $\mathrm{~m}$ & & 5.69 & $\mathrm{dt}$ & $15.4,7.7,7.7$ \\
\hline $15 \mathrm{a}$ & 2.77 & $\mathrm{dd}$ & $13.9,3.6$ & 2.76 & $\mathrm{~s}$ br $(2 \mathrm{H})$ & \\
\hline $15 \mathrm{~b}$ & 2.67 & $\mathrm{dd}$ & $13.9,10.3$ & 2.76 & $\mathrm{~s}$ br $(2 \mathrm{H})$ & \\
\hline $17 \mathrm{a}$ & 2.53 & $\mathrm{dd}$ & $12.9,3.1$ & 2.35 & dd & $14.0,5.5$ \\
\hline $17 \mathrm{~b}$ & 1.78 & $\mathrm{dd}$ & $12.9,10.8$ & 1.92 & $\mathrm{dd}$ & $14.0,9.5$ \\
\hline 18 & $2.18-2.13$ & $\mathrm{~m}$ & & 2.17 & $\mathrm{~m}$ & \\
\hline 19 & 4.55 & dd & $7.1,3.8$ & 4.72 & $\mathrm{dd}$ & $5.9,3.4$ \\
\hline 20 & 2.92 & $\mathrm{dd}$ & $7.1,2.2$ & 2.85 & $\mathrm{dd}$ & $5.9,1.9$ \\
\hline 21 & 2.70 & $\mathrm{dd}$ & $7.5,2.2$ & 2.76 & $\mathrm{dd}$ & 1.9 \\
\hline 22 & $1.70-1.26$ & $\mathrm{~m}(5 \mathrm{H})$ & & 1.38 & $\mathrm{~m}$ & \\
\hline 23 & $1.70-1.26$ & $\mathrm{~m}(5 \mathrm{H})$ & & $1.55-1.25$ & $\mathrm{~m}(4 \mathrm{H})$ & \\
\hline 24 & $1.70-1.26$ & $\mathrm{~m}(5 \mathrm{H})$ & & $1.55-1.25$ & $\mathrm{~m}(4 \mathrm{H})$ & \\
\hline $25(3 \mathrm{H})$ & 0.92 & $\mathrm{t}$ & 7.1 & 0.91 & $\mathrm{t}$ & 6.8 \\
\hline $26(3 \mathrm{H})$ & 2.30 & $\mathrm{~s}$ & - & 2.27 & $\mathrm{~s}$ & - \\
\hline $27 \mathrm{a}$ & 5.45 & $\mathrm{~s}$ & - & 5.36 & $\mathrm{~s}$ & - \\
\hline $27 \mathrm{~b}$ & 5.23 & $\mathrm{~s}$ & - & 5.21 & $\mathrm{~s}$ & - \\
\hline $28 \mathrm{a}$ & 5.59 & $\mathrm{~s}$ & - & 5.49 & $\mathrm{~s}$ & - \\
\hline $28 b$ & 5.58 & $\mathrm{~s}$ & - & 5.37 & $\mathrm{~s}$ & - \\
\hline $29 a$ & 4.87 & $\mathrm{~s}$ & - & 4.88 & $\mathrm{~s}$ & - \\
\hline $29 b$ & 4.78 & $\mathrm{~s}$ & - & 4.79 & $\mathrm{~s}$ & - \\
\hline $30(3 \mathrm{H})$ & 0.99 & $\mathrm{~d}$ & 7.1 & 1.06 & $\mathrm{~d}$ & 7.0 \\
\hline $31(3 \mathrm{H})$ & 0.98 & $\mathrm{~d}$ & 6.8 & 0.93 & $\mathrm{~d}$ & 6.0 \\
\hline
\end{tabular}


${ }^{1} \mathrm{H}$ NMR data for 36 in $\mathrm{CDCl}_{3}$.

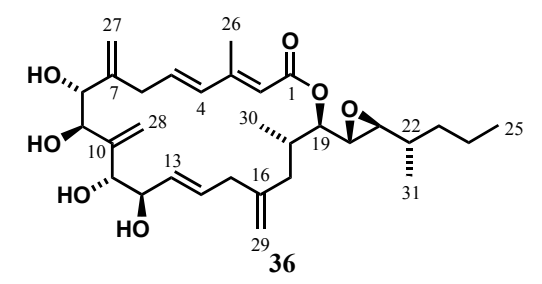

\begin{tabular}{|c|c|c|c|c|c|c|}
\hline & $\underline{\text { Isomer } 36}$ & & & $\underline{\text { Natural Product }}$ & & \\
\hline Proton \# & $\delta_{\mathrm{H}}$ & Mult. & $J$ & $\delta_{\mathrm{H}}$ & Mult. & $J$ \\
\hline 2 & 5.80 & $\mathrm{~s}$ & - & 5.80 & $\mathrm{~s}$ & - \\
\hline 4 & 6.14 & $\mathrm{~d}$ & 15.6 & 6.27 & $\mathrm{~d}$ & 15.6 \\
\hline 5 & 6.06 & $\mathrm{dt}$ & $15.4,6.7,6.7$ & 6.09 & ddd & $15.6,8.8,4.8$ \\
\hline $6 \mathrm{a}$ & 3.19 & $\mathrm{dd}$ & $14.4,6.7$ & 3.20 & $\mathrm{dd}$ & $14.6,4.8$ \\
\hline $6 b$ & 2.96 & $\mathrm{dd}$ & $14.1,7.2$ & 3.13 & $\mathrm{dd}$ & $14.6,8.8$ \\
\hline 8 & 4.09 & $\mathrm{~d}$ & 4.6 & 4.42 & $\mathrm{sbr}$ & - \\
\hline 9 & 4.37 & $\mathrm{~d}$ & 4.6 & 4.58 & $\mathrm{~s} b r$ & - \\
\hline 11 & 3.88 & $\mathrm{~d}$ & 3.3 & 4.09 & $\mathrm{sbr}$ & - \\
\hline 12 & 4.16 & $\mathrm{t}$ & $4.4,4.4$ & 4.22 & $\mathrm{~s} b r$ & - \\
\hline 13 & 5.36 & $\mathrm{dd}$ & $15.4,4.6$ & 5.51 & $\mathrm{dd}$ & $15.4,4.1$ \\
\hline 14 & 5.67 & $\mathrm{dt}$ & $14.1,6.4,6.4$ & 5.69 & $\mathrm{dt}$ & $15.4,7.7,7.7$ \\
\hline $15 \mathrm{a}$ & 2.71 & $\mathrm{dd}$ & $14.1,6.7$ & 2.76 & $\mathrm{~s}$ br $(2 \mathrm{H})$ & \\
\hline $15 b$ & 2.61 & $\mathrm{dd}$ & $14.1,8.0$ & 2.76 & $\mathrm{~s}$ br $(2 \mathrm{H})$ & \\
\hline $17 \mathrm{a}$ & 2.41 & $\mathrm{dd}$ & $14.1,3.6$ & 2.35 & $\mathrm{dd}$ & $14.0,5.5$ \\
\hline 19 & 4.58 & $\mathrm{dd}$ & $6.9,3.3$ & 4.72 & $\mathrm{dd}$ & $5.9,3.4$ \\
\hline 20 & 2.90 & $\mathrm{dd}$ & $6.7,3.1$ & 2.85 & $\mathrm{dd}$ & $5.9,1.9$ \\
\hline 21 & 2.66 & $\mathrm{dd}$ & $7.7,2.1$ & 2.76 & $\mathrm{dd}$ & 1.9 \\
\hline $26(3 \mathrm{H})$ & 2.23 & $\mathrm{~s}$ & - & 2.27 & $\mathrm{~s}$ & - \\
\hline $27 \mathrm{a}$ & 5.30 & $\mathrm{~s}$ & - & 5.36 & $\mathrm{~s}$ & - \\
\hline $27 \mathrm{~b}$ & 5.18 & $\mathrm{~s}$ & - & 5.21 & $\mathrm{~s}$ & - \\
\hline $28 \mathrm{a}$ & 5.46 & $\mathrm{~s}$ & - & 5.49 & $\mathrm{~s}$ & - \\
\hline $28 \mathrm{~b}$ & 5.37 & $\mathrm{~s}$ & - & 5.37 & $\mathrm{~s}$ & - \\
\hline $29 a$ & 4.83 & $\mathrm{~s}$ & - & 4.88 & $\mathrm{~s}$ & - \\
\hline $29 b$ & 4.75 & $\mathrm{~s}$ & - & 4.79 & $\mathrm{~s}$ & - \\
\hline
\end{tabular}


${ }^{1} \mathrm{H}$ NMR data for 38 in $\mathrm{CDCl}_{3}$.

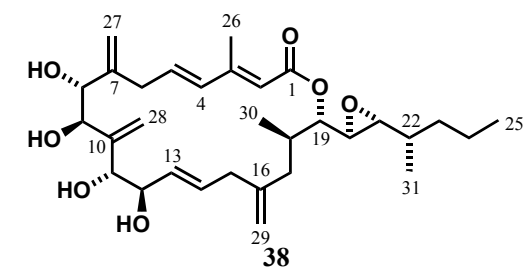

\begin{tabular}{|c|c|c|c|c|c|c|}
\hline \multirow[b]{2}{*}{ Proton \# } & \multicolumn{3}{|l|}{$\underline{\text { Isomer } 38}$} & \multicolumn{3}{|c|}{$\underline{\text { Natural Product }}$} \\
\hline & $\delta_{\mathrm{H}}$ & Mult. & $J$ & $\delta_{\mathrm{H}}$ & Mult. & $J$ \\
\hline 2 & 5.79 & $\mathrm{~s}$ & - & 5.80 & $\mathrm{~s}$ & - \\
\hline 4 & 6.19 & $\mathrm{~d}$ & 15.6 & 6.27 & $\mathrm{~d}$ & 15.6 \\
\hline 5 & 6.08 & ddd & $14.9,9.8,3.8$ & 6.09 & ddd & $15.6,8.8,4.8$ \\
\hline $6 a$ & 3.22 & $\mathrm{dd}$ & $15.1,4.4$ & 3.20 & $\mathrm{dd}$ & $14.6,4.8$ \\
\hline $6 b$ & 3.11 & $\mathrm{dd}$ & $15.3,10.4$ & 3.13 & $\mathrm{dd}$ & $14.6,8.8$ \\
\hline 8 & 4.23 & $\mathrm{~s} \mathrm{br}$ & - & 4.42 & $\mathrm{~s}$ br & - \\
\hline 9 & 4.28 & $\mathrm{~s}$ br & - & 4.58 & $\mathrm{~s}$ br & - \\
\hline 11 & 3.83 & $\mathrm{~s}$ br & - & 4.09 & $\mathrm{~s}$ br & - \\
\hline 12 & 4.20 & $\mathrm{~s}$ br & - & 4.22 & $\mathrm{~s}$ br & - \\
\hline 13 & 5.26 & $\mathrm{~d} \mathrm{br}$ & 16.2 & 5.51 & dd & $15.4,4.1$ \\
\hline 14 & $5.77-5.72$ & $\mathrm{~m}$ & & 5.69 & $\mathrm{dt}$ & $15.4,7.7,7.7$ \\
\hline $15 \mathrm{a}$ & 2.76 & $\mathrm{dd}$ & $13.6,4.0$ & 2.76 & $\mathrm{~s}$ br $(2 \mathrm{H})$ & \\
\hline $15 b$ & 2.66 & dd & $14.2,10.4$ & 2.76 & $\mathrm{~s}$ br $(2 \mathrm{H})$ & \\
\hline $17 \mathrm{a}$ & 2.51 & dd & $12.5,2.5$ & 2.35 & $\mathrm{dd}$ & $14.0,5.5$ \\
\hline $17 b$ & 1.79 & $\mathrm{dd}$ & $12.7,11.6$ & 1.92 & $\mathrm{dd}$ & $14.0,9.5$ \\
\hline 18 & 2.13 & $\mathrm{~m}$ & & 2.17 & $\mathrm{~m}$ & \\
\hline 19 & 4.61 & $\mathrm{dd}$ & $6.2,3.8$ & 4.72 & $\mathrm{dd}$ & $5.9,3.4$ \\
\hline 20 & 2.97 & $\mathrm{dd}$ & $6.1,2.1$ & 2.85 & $\mathrm{dd}$ & $5.9,1.9$ \\
\hline 21 & 2.69 & dd & $8.9,2.1$ & 2.76 & dd & 1.9 \\
\hline 22 & $1.44-1.22$ & $\mathrm{~m}(5 \mathrm{H})$ & & 1.38 & $\mathrm{~m}$ & \\
\hline 23 & $1.44-1.22$ & $\mathrm{~m}(5 \mathrm{H})$ & & $1.55-1.25$ & $\mathrm{~m}(4 \mathrm{H})$ & \\
\hline 24 & $1.44-1.22$ & $\mathrm{~m}(5 \mathrm{H})$ & & $1.55-1.25$ & $\mathrm{~m}(4 \mathrm{H})$ & \\
\hline $25(3 \mathrm{H})$ & 0.91 & $\mathrm{t}$ & 7.0 & 0.91 & $\mathrm{t}$ & 6.8 \\
\hline $26(3 \mathrm{H})$ & 2.29 & $\mathrm{~s}$ & - & 2.27 & $\mathrm{~s}$ & - \\
\hline $27 \mathrm{a}$ & 5.37 & $\mathrm{~s}$ & - & 5.36 & $\mathrm{~s}$ & - \\
\hline $27 b$ & 5.22 & $\mathrm{~s}$ & - & 5.21 & $\mathrm{~s}$ & - \\
\hline $28 \mathrm{a}$ & 5.59 & $\mathrm{~s}$ & - & 5.49 & $\mathrm{~s}$ & - \\
\hline $28 b$ & 5.57 & $\mathrm{~s}$ & - & 5.37 & $\mathrm{~s}$ & - \\
\hline $29 \mathrm{a}$ & 4.86 & $\mathrm{~s}$ & - & 4.88 & $\mathrm{~s}$ & - \\
\hline $29 b$ & 4.78 & $\mathrm{~s}$ & - & 4.79 & $\mathrm{~s}$ & - \\
\hline $30(3 \mathrm{H})$ & 0.99 & $\mathrm{~d}$ & 7.0 & 1.06 & $\mathrm{~d}$ & 7.0 \\
\hline $31(3 \mathrm{H})$ & 1.01 & $\mathrm{~d}$ & 6.4 & 0.93 & $\mathrm{~d}$ & 6.0 \\
\hline
\end{tabular}


$\mathrm{H}$ NMR data for 39 in $\mathrm{CDCl}_{3}$.

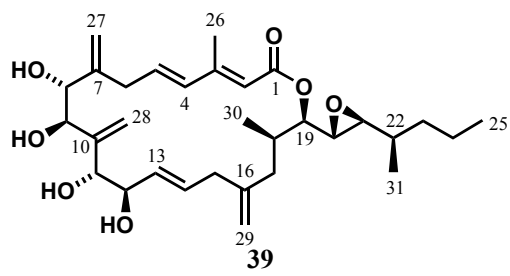

Proton \#

$$
\delta_{\mathrm{H}}
$$

Mult. $J$

Natural Product

$\delta_{\mathrm{H}} \quad$ Mult. $J$

\begin{tabular}{|c|c|c|c|c|c|c|}
\hline 2 & 5.81 & $\mathrm{~s}$ & - & 5.80 & $\mathrm{~s}$ & - \\
\hline 4 & 6.19 & d & 15.4 & 6.27 & d & 15.6 \\
\hline 5 & 6.08 & ddd & $14.7,8.1,6.4$ & 6.09 & ddd & $15.6,8.8,4.8$ \\
\hline $6 \mathrm{a}$ & 3.25 & $\mathrm{dd}$ & $14.4,6.4$ & 3.20 & $\mathrm{dd}$ & $14.6,4.8$ \\
\hline $6 \mathrm{~b}$ & 3.00 & $\mathrm{dd}$ & $14.4,8.2$ & 3.13 & $\mathrm{dd}$ & $14.6,8.8$ \\
\hline 8 & 4.32 & $\mathrm{~s}$ br & - & 4.42 & $\mathrm{~s} b r$ & - \\
\hline 9 & 4.16 & $\mathrm{~s}$ br & - & 4.58 & $\mathrm{~s}$ br & - \\
\hline 11 & 3.95 & $\mathrm{~s}$ br & - & 4.09 & $\mathrm{~s} b r$ & - \\
\hline 12 & 4.09 & $\mathrm{~m}$ & - & 4.22 & $\mathrm{~s}$ br & - \\
\hline 13 & 5.48 & $\mathrm{ddm}$ & $15.6,4.9$ & 5.51 & dd & $15.4,4.1$ \\
\hline 14 & 5.72 & dddd & $15.4,8.6,7.0,1.2$ & 5.69 & $\mathrm{dt}$ & $15.4,7.7,7.7$ \\
\hline $15 \mathrm{a}$ & $2.76-2.74$ & $\mathrm{~m}(2 \mathrm{H})$ & & 2.76 & $\mathrm{~s} \mathrm{br}(2 \mathrm{H})$ & \\
\hline $15 \mathrm{~b}$ & $2.76-2.74$ & $\mathrm{~m}(2 \mathrm{H})$ & & 2.76 & $\mathrm{~s} \mathrm{br}(2 \mathrm{H})$ & \\
\hline $17 \mathrm{a}$ & 2.17 & $\mathrm{dd}$ & $12.9,2.6$ & 2.35 & dd & $14.0,5.5$ \\
\hline $17 \mathrm{~b}$ & 1.65 & $\mathrm{dd}$ & $13.3,9.6$ & 1.92 & dd & $14.0,9.5$ \\
\hline 18 & 1.94 & $\mathrm{~m}$ & & 2.17 & $\mathrm{~m}$ & \\
\hline 19 & 4.60 & $\mathrm{dd}$ & $10.3,5.5$ & 4.72 & $\mathrm{dd}$ & $5.9,3.4$ \\
\hline 20 & 3.02 & $\mathrm{dd}$ & $5.6,2.2$ & 2.85 & dd & $5.9,1.9$ \\
\hline 21 & 2.72 & $\mathrm{dd}$ & $6.7,2.1$ & 2.76 & $\mathrm{dd}$ & 1.9 \\
\hline 22 & $1.47-1.21$ & $\mathrm{~m}(5 \mathrm{H})$ & & 1.38 & $\mathrm{~m}$ & \\
\hline 23 & $1.47-1.21$ & $\mathrm{~m}(5 \mathrm{H})$ & & $1.55-1.25$ & $\mathrm{~m}(4 \mathrm{H})$ & \\
\hline 24 & $1.47-1.21$ & $\mathrm{~m}(5 \mathrm{H})$ & & $1.55-1.25$ & $\mathrm{~m}(4 \mathrm{H})$ & \\
\hline $25(3 \mathrm{H})$ & 0.92 & $\mathrm{t}$ & 7.2 & 0.91 & $\mathrm{t}$ & 6.8 \\
\hline $26(3 \mathrm{H})$ & 2.26 & d & 1.0 & 2.27 & $\mathrm{~s}$ & - \\
\hline $27 \mathrm{a}$ & 5.34 & $\mathrm{~s}$ & - & 5.36 & $\mathrm{~s}$ & - \\
\hline $27 \mathrm{~b}$ & 5.24 & $\mathrm{~s}$ & - & 5.21 & $\mathrm{~s}$ & - \\
\hline $28 \mathrm{a}$ & 5.49 & $\mathrm{~s}$ & - & 5.49 & $\mathrm{~s}$ & - \\
\hline $28 \mathrm{~b}$ & 5.44 & $\mathrm{~s}$ & - & 5.37 & $\mathrm{~s}$ & - \\
\hline $29 a$ & 4.83 & $\mathrm{~s}$ & - & 4.88 & $\mathrm{~s}$ & - \\
\hline $29 b$ & 4.75 & $\mathrm{~s}$ & - & 4.79 & $\mathrm{~s}$ & - \\
\hline $30(3 \mathrm{H})$ & 1.04 & d & 6.7 & 1.06 & $d$ & 7.0 \\
\hline $31(3 \mathrm{H})$ & 1.00 & d & 6.6 & 0.93 & d & 6.0 \\
\hline
\end{tabular}


${ }^{1} \mathrm{H}$ NMR data for $\mathbf{4 0}$ in $\mathrm{CDCl}_{3}$.

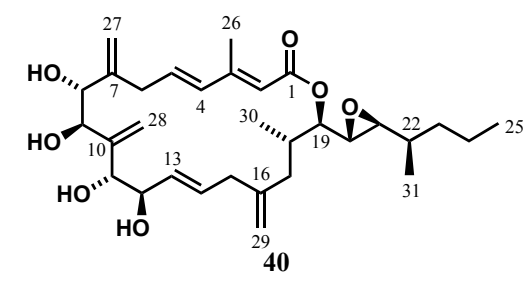

\begin{tabular}{|c|c|c|c|c|c|c|}
\hline & Isomer 40 & & & $\underline{\text { Natural Product }}$ & & \\
\hline Proton \# & $\delta_{\mathrm{H}}$ & Mult. & $J$ & $\delta_{\mathrm{H}}$ & Mult. & $J$ \\
\hline 2 & 5.82 & $\mathrm{~s}$ & - & 5.80 & $\mathrm{~s}$ & - \\
\hline 4 & 6.16 & $\mathrm{~d}$ & 15.9 & 6.27 & $\mathrm{~d}$ & 15.6 \\
\hline 5 & 6.08 & ddd & $15.5,7.0,6.7$ & 6.09 & ddd & $15.6,8.8,4.8$ \\
\hline $6 a$ & 3.21 & $\mathrm{dd}$ & $14.7,6.5$ & 3.20 & $\mathrm{dd}$ & $14.6,4.8$ \\
\hline $6 \mathrm{~b}$ & 2.98 & $\mathrm{dd}$ & $14.5,7.4$ & 3.13 & $\mathrm{dd}$ & $14.6,8.8$ \\
\hline 8 & 4.38 & $\mathrm{~d}$ & 4.3 & 4.42 & $\mathrm{~s} b r$ & - \\
\hline 9 & 4.10 & $\mathrm{~d}$ & 4.3 & 4.58 & $\mathrm{sbr}$ & - \\
\hline 11 & 3.89 & $d$ & 3.5 & 4.09 & $\mathrm{~s} b r$ & - \\
\hline 12 & 4.17 & $\mathrm{dd}$ & $4.0,3.7$ & 4.22 & $\mathrm{~s}$ br & - \\
\hline 13 & 5.38 & $\mathrm{dd}$ & $15.4,5.4$ & 5.51 & $\mathrm{dd}$ & $15.4,4.1$ \\
\hline 14 & 5.68 & dddd & $15.4,7.8,6.6,1.2$ & 5.69 & $\mathrm{dt}$ & $15.4,7.7,7.7$ \\
\hline $15 \mathrm{a}$ & 2.73 & $\mathrm{dd}$ & $13.9,6.0$ & 2.76 & $\mathrm{~s}$ br $(2 \mathrm{H})$ & \\
\hline $15 \mathrm{~b}$ & 2.63 & $\mathrm{dd}$ & $14.7,8.3$ & 2.76 & $\mathrm{~s}$ br $(2 \mathrm{H})$ & \\
\hline $17 \mathrm{a}$ & 2.40 & $\mathrm{dd}$ & $13.7,3.9$ & 2.35 & $\mathrm{dd}$ & $14.0,5.5$ \\
\hline $17 \mathrm{~b}$ & 1.82 & $\mathrm{dd}$ & $13.7,10.1$ & 1.92 & $\mathrm{dd}$ & $14.0,9.5$ \\
\hline 18 & 2.11 & $\mathrm{~m}$ & & 2.17 & $\mathrm{~m}$ & \\
\hline 19 & 4.67 & $\mathrm{dd}$ & $6.1,3.4$ & 4.72 & $\mathrm{dd}$ & $5.9,3.4$ \\
\hline 20 & 2.97 & $\mathrm{dd}$ & $6.1,2.2$ & 2.85 & $\mathrm{dd}$ & $5.9,1.9$ \\
\hline 21 & 2.66 & $\mathrm{dd}$ & $7.0,2.2$ & 2.76 & $\mathrm{dd}$ & 1.9 \\
\hline 22 & $1.43-1.20$ & $\mathrm{~m}(5 \mathrm{H})$ & & 1.38 & $\mathrm{~m}$ & \\
\hline 23 & $1.43-1.20$ & $\mathrm{~m}(5 \mathrm{H})$ & & $1.55-1.25$ & $\mathrm{~m}(4 \mathrm{H})$ & \\
\hline 24 & $1.43-1.20$ & $\mathrm{~m}(5 \mathrm{H})$ & & $1.55-1.25$ & $\mathrm{~m}(4 \mathrm{H})$ & \\
\hline $25(3 \mathrm{H})$ & 0.91 & $\mathrm{t}$ & 7.0 & 0.91 & $\mathrm{t}$ & 6.8 \\
\hline $26(3 \mathrm{H})$ & 2.25 & $d$ & 1.1 & 2.27 & $\mathrm{~s}$ & - \\
\hline $27 \mathrm{a}$ & 5.32 & $\mathrm{~s}$ & - & 5.36 & $\mathrm{~s}$ & - \\
\hline $27 \mathrm{~b}$ & 5.20 & $\mathrm{~s}$ & - & 5.21 & $\mathrm{~s}$ & - \\
\hline $28 \mathrm{a}$ & 5.47 & $\mathrm{~s}$ & - & 5.49 & $\mathrm{~s}$ & - \\
\hline $28 \mathrm{~b}$ & 5.39 & $\mathrm{~s}$ & - & 5.37 & $\mathrm{~s}$ & - \\
\hline $29 a$ & 4.85 & $\mathrm{~s}$ & - & 4.88 & $\mathrm{~s}$ & - \\
\hline $29 \mathrm{~b}$ & 4.77 & $\mathrm{~s}$ & - & 4.79 & $\mathrm{~s}$ & - \\
\hline $30(3 \mathrm{H})$ & 1.01 & $d$ & 7.0 & 1.06 & d & 7.0 \\
\hline $31(3 \mathrm{H})$ & 1.00 & $\mathrm{~d}$ & 6.5 & 0.93 & $\mathrm{~d}$ & 6.0 \\
\hline
\end{tabular}


${ }^{1} \mathrm{H}$ NMR data for 54 in $\mathrm{CDCl}_{3}$.

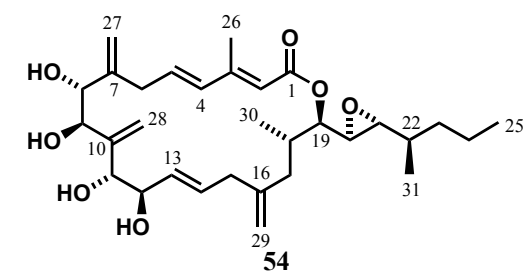

Isomer 54

Natural Product

Proton \#

$\delta_{\mathrm{H}}$

Mult. J

$\delta_{\mathrm{H}}$

Mult. $\quad J$

\begin{tabular}{|c|c|c|c|c|c|c|}
\hline 2 & 5.77 & $\mathrm{~s}$ & - & 5.80 & $\mathrm{~s}$ & - \\
\hline 4 & 6.16 & $\mathrm{~d}$ & 15.6 & 6.27 & d & 15.6 \\
\hline 5 & 6.08 & ddd & $15.9,6.8,6.5$ & 6.09 & ddd & $15.6,8.8,4.8$ \\
\hline $6 a$ & 3.21 & $\mathrm{dd}$ & $14.9,7.0$ & 3.20 & $\mathrm{dd}$ & $14.6,4.8$ \\
\hline $6 b$ & 2.98 & $\mathrm{dd}$ & $14.4,7.1$ & 3.13 & dd & $14.6,8.8$ \\
\hline 8 & 4.38 & $\mathrm{~s}$ br & - & 4.42 & $\mathrm{~s}$ br & - \\
\hline 9 & 4.12 & $\mathrm{~s}$ br & - & 4.58 & $\mathrm{~s}$ br & - \\
\hline 11 & 3.91 & $\mathrm{~s}$ br & - & 4.09 & $\mathrm{~s}$ br & - \\
\hline 12 & 4.18 & $\mathrm{~s} b r$ & - & 4.22 & $\mathrm{~s}$ br & - \\
\hline 13 & 5.41 & $\mathrm{dd}$ & $15.5,5.4$ & 5.51 & dd & $15.4,4.1$ \\
\hline 14 & 5.69 & ddd & $14.2,6.6,6.3$ & 5.69 & $\mathrm{dt}$ & $15.4,7.7,7.7$ \\
\hline $15 \mathrm{a}$ & 2.74 & $\mathrm{dd}$ & $14.8,6.7$ & 2.76 & $\mathrm{~s}$ br $(2 \mathrm{H})$ & \\
\hline $15 b$ & 2.66 & $\mathrm{dd}$ & $14.8,7.3$ & 2.76 & $\mathrm{~s}$ br $(2 \mathrm{H})$ & \\
\hline $17 \mathrm{a}$ & 2.26 & $\mathrm{~d} b r$ & 14.0 & 2.35 & $\mathrm{dd}$ & $14.0,5.5$ \\
\hline $17 \mathrm{~b}$ & 1.88 & $\mathrm{dd}$ & $13.9,9.2$ & 1.92 & dd & $14.0,9.5$ \\
\hline 18 & 2.14 & $\mathrm{~m}$ & & 2.17 & $\mathrm{~m}$ & \\
\hline 19 & 4.67 & $\mathrm{dd}$ & $6.4,3.2$ & 4.72 & $\mathrm{dd}$ & $5.9,3.4$ \\
\hline 20 & 2.84 & dd & $6.2,2.1$ & 2.85 & $\mathrm{dd}$ & $5.9,1.9$ \\
\hline 21 & 2.77 & $\mathrm{dd}$ & $7.6,2.2$ & 2.76 & $\mathrm{dd}$ & 1.9 \\
\hline 22 & $1.51-1.25$ & $\mathrm{~m}(5 \mathrm{H})$ & & 1.38 & $\mathrm{~m}$ & \\
\hline 23 & $1.51-1.25$ & $\mathrm{~m}(5 \mathrm{H})$ & & $1.55-1.25$ & $\mathrm{~m}(4 \mathrm{H})$ & \\
\hline 24 & $1.51-1.25$ & $\mathrm{~m}(5 \mathrm{H})$ & & $1.55-1.25$ & $\mathrm{~m}(4 \mathrm{H})$ & \\
\hline $25(3 \mathrm{H})$ & 0.91 & $\mathrm{t}$ & 7.0 & 0.91 & $\mathrm{t}$ & 6.8 \\
\hline $26(3 \mathrm{H})$ & 2.24 & $\mathrm{~d}$ & 1.0 & 2.27 & $\mathrm{~s}$ & - \\
\hline $27 \mathrm{a}$ & 5.33 & $\mathrm{~s}$ & - & 5.36 & $\mathrm{~s}$ & - \\
\hline $27 \mathrm{~b}$ & 5.21 & $\mathrm{~s}$ & - & 5.21 & $\mathrm{~s}$ & - \\
\hline $28 \mathrm{a}$ & 5.48 & $\mathrm{~s}$ & - & 5.49 & $\mathrm{~s}$ & - \\
\hline $28 \mathrm{~b}$ & 5.39 & $\mathrm{~s}$ & - & 5.37 & $\mathrm{~s}$ & - \\
\hline $29 a$ & 4.87 & $\mathrm{~s}$ & - & 4.88 & $\mathrm{~s}$ & - \\
\hline $29 b$ & 4.79 & $\mathrm{~s}$ & - & 4.79 & $\mathrm{~S}$ & - \\
\hline $30(3 \mathrm{H})$ & 1.05 & $\mathrm{~d}$ & 7.1 & 1.06 & $\mathrm{~d}$ & 7.0 \\
\hline $31(3 \mathrm{H})$ & 0.89 & $\mathrm{~d}$ & 7.1 & 0.93 & $\mathrm{~d}$ & 6.0 \\
\hline
\end{tabular}


${ }^{1} \mathrm{H}$ NMR data for $\mathbf{4 1}$ in $\mathrm{CDCl}_{3}$.

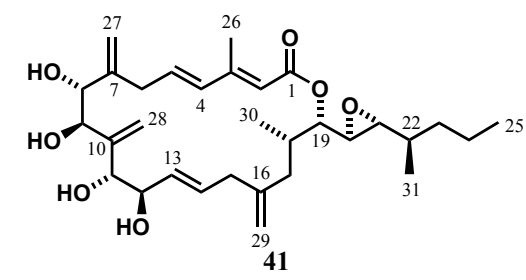

$\underline{\text { Isomer } 41}$

\begin{tabular}{|c|c|c|c|c|c|c|}
\hline Proton \# & $\delta_{\mathrm{H}}$ & Mult. & $J$ & $\delta_{\mathrm{H}}$ & Mult. & $J$ \\
\hline 2 & 5.72 & $\mathrm{~s}$ & - & 5.80 & $\mathrm{~s}$ & - \\
\hline 4 & 6.16 & $\mathrm{~d}$ & 15.6 & 6.27 & $\mathrm{~d}$ & 15.6 \\
\hline 5 & 6.03 & ddd & $14.9,9.8,4.8$ & 6.09 & ddd & $15.6,8.8,4.8$ \\
\hline $6 a$ & 3.16 & $\mathrm{dd}$ & $14.4,4.3$ & 3.20 & $\mathrm{dd}$ & $14.6,4.8$ \\
\hline $6 \mathrm{~b}$ & 3.04 & $\mathrm{dd}$ & $14.7,9.9$ & 3.13 & $\mathrm{dd}$ & $14.6,8.8$ \\
\hline 8 & 4.36 & $\mathrm{~s} b r$ & - & 4.42 & $\mathrm{~s} b r$ & - \\
\hline 9 & 4.30 & $\mathrm{~s} b r$ & - & 4.58 & $\mathrm{~s} b r$ & - \\
\hline 11 & 3.99 & $\mathrm{~s} b r$ & - & 4.09 & $\mathrm{~s} b r$ & - \\
\hline 12 & 4.27 & $\mathrm{~s} b r$ & - & 4.22 & $\mathrm{~s} b r$ & - \\
\hline 13 & 5.53 & $\mathrm{dd}$ & $15.6,5.4$ & 5.51 & $\mathrm{dd}$ & $15.4,4.1$ \\
\hline 14 & 5.75 & ddd & $15.3,7.3,6.4$ & 5.69 & $\mathrm{dt}$ & $15.4,7.7,7.7$ \\
\hline $15 \mathrm{a}$ & 2.81 & $\mathrm{dd}$ & $15.3,7.0$ & 2.76 & $\mathrm{~s} b \mathrm{br}(2 \mathrm{H})$ & \\
\hline $15 \mathrm{~b}$ & 2.68 & $\mathrm{~m}$ & & 2.76 & $\mathrm{~s}$ br $(2 \mathrm{H})$ & \\
\hline $17 \mathrm{a}$ & 2.15 & $\mathrm{dd}$ & $13.8,4.8$ & 2.35 & $\mathrm{dd}$ & $14.0,5.5$ \\
\hline $17 \mathrm{~b}$ & 1.76 & $\mathrm{dd}$ & $13.9,8.4$ & 1.92 & $\mathrm{dd}$ & $14.0,9.5$ \\
\hline 18 & 2.01 & $\mathrm{~m}$ & & 2.17 & $\mathrm{~m}$ & \\
\hline 19 & 4.56 & $\mathrm{dd}$ & $10.3,5.6$ & 4.72 & $\mathrm{dd}$ & $5.9,3.4$ \\
\hline 20 & 2.91 & $\mathrm{dd}$ & $5.7,2.1$ & 2.85 & $\mathrm{dd}$ & $5.9,1.9$ \\
\hline 21 & 2.70 & $\mathrm{dd}$ & $7.6,2.0$ & 2.76 & $\mathrm{dd}$ & 1.9 \\
\hline 22 & $1.51-1.19$ & $\mathrm{~m}(5 \mathrm{H})$ & & 1.38 & $\mathrm{~m}$ & \\
\hline 23 & $1.51-1.19$ & $\mathrm{~m}(5 \mathrm{H})$ & & $1.55-1.25$ & $\mathrm{~m}(4 \mathrm{H})$ & \\
\hline 24 & $1.51-1.19$ & $\mathrm{~m}(5 \mathrm{H})$ & & $1.55-1.25$ & $\mathrm{~m}(4 \mathrm{H})$ & \\
\hline $25(3 \mathrm{H})$ & 0.89 & $\mathrm{t}$ & 7.2 & 0.91 & $\mathrm{t}$ & 6.8 \\
\hline $26(3 \mathrm{H})$ & 2.24 & $\mathrm{~s}$ & - & 2.27 & $\mathrm{~s}$ & - \\
\hline $27 \mathrm{a}$ & 5.33 & $\mathrm{~s}$ & - & 5.36 & $\mathrm{~s}$ & - \\
\hline $27 \mathrm{~b}$ & 5.20 & $\mathrm{~s}$ & - & 5.21 & $\mathrm{~s}$ & - \\
\hline $28 \mathrm{a}$ & 5.49 & $\mathrm{~s}$ & - & 5.49 & $\mathrm{~s}$ & - \\
\hline $28 \mathrm{~b}$ & 5.42 & $\mathrm{~s}$ & - & 5.37 & $\mathrm{~s}$ & - \\
\hline $29 a$ & 4.74 & $\mathrm{~s}$ & - & 4.88 & $\mathrm{~s}$ & - \\
\hline $29 \mathrm{~b}$ & 4.73 & $\mathrm{~s}$ & - & 4.79 & $\mathrm{~s}$ & - \\
\hline $30(3 \mathrm{H})$ & 1.02 & $\mathrm{~d}$ & 6.7 & 1.06 & $d$ & 7.0 \\
\hline $31(3 \mathrm{H})$ & 0.93 & $\mathrm{~d}$ & 6.8 & 0.93 & $\mathrm{~d}$ & 6.0 \\
\hline
\end{tabular}


${ }^{1} \mathrm{H}$ NMR data for $\mathbf{5 6}$ in $\mathrm{CDCl}_{3}$.

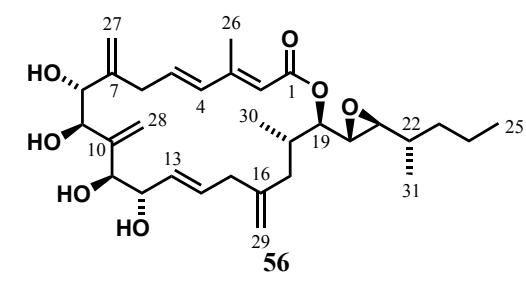

Isomer 56

Natural Product

Proton \#

$\delta_{\mathrm{H}}$

Mult. J

$\delta_{\mathrm{H}}$

Mult. $\quad J$

\begin{tabular}{|c|c|c|c|c|c|c|}
\hline 2 & 5.79 & $\mathrm{~s}$ & - & 5.80 & $\mathrm{~s}$ & - \\
\hline 4 & 6.20 & $\mathrm{~d}$ & 15.8 & 6.27 & $d$ & 15.6 \\
\hline 5 & 6.08 & ddd & $14.5,8.4,5.6$ & 6.09 & ddd & $15.6,8.8,4.8$ \\
\hline $6 a$ & 3.22 & $\mathrm{dd}$ & $14.5,5.4$ & 3.20 & $\mathrm{dd}$ & $14.6,4.8$ \\
\hline $6 b$ & 2.94 & dd & $13.1,8.9$ & 3.13 & dd & $14.6,8.8$ \\
\hline 8 & 4.42 & $\mathrm{~d}$ & 3.2 & 4.42 & $\mathrm{~s}$ br & - \\
\hline 9 & 4.28 & $\mathrm{~d}$ & 3.2 & 4.58 & $\mathrm{~s}$ br & - \\
\hline 11 & 4.02 & $\mathrm{~d}$ & 3.9 & 4.09 & $\mathrm{~s}$ br & - \\
\hline 12 & 4.17 & $\mathrm{t}$ & $4.5,4.5$ & 4.22 & $\mathrm{~s}$ br & - \\
\hline 13 & 5.42 & $\mathrm{dd}$ & $15.4,5.4$ & 5.51 & $\mathrm{dd}$ & $15.4,4.1$ \\
\hline 14 & 5.68 & dddd & $15.0,8.3,7.1,1.3$ & 5.69 & $\mathrm{dt}$ & $15.4,7.7,7.7$ \\
\hline $15 \mathrm{a}$ & 2.74 & $\mathrm{dd}$ & $14.6,5.1$ & 2.76 & $\mathrm{~s}$ br $(2 \mathrm{H})$ & \\
\hline $15 b$ & 2.67 & $\mathrm{dd}$ & $14.4,7.7$ & 2.76 & $\mathrm{~s}$ br $(2 \mathrm{H})$ & \\
\hline $17 \mathrm{a}$ & 2.50 & $\mathrm{dd}$ & $13.3,3.4$ & 2.35 & $\mathrm{dd}$ & $14.0,5.5$ \\
\hline $17 \mathrm{~b}$ & 1.81 & dd & $13.4,10.6$ & 1.92 & dd & $14.0,9.5$ \\
\hline 18 & 2.10 & $\mathrm{~m}$ & & 2.17 & $\mathrm{~m}$ & \\
\hline 19 & 4.62 & $\mathrm{dd}$ & $6.7,3.5$ & 4.72 & $\mathrm{dd}$ & $5.9,3.4$ \\
\hline 20 & 2.92 & $\mathrm{dd}$ & $7.0,2.3$ & 2.85 & $\mathrm{dd}$ & $5.9,1.9$ \\
\hline 21 & 2.68 & $\mathrm{dd}$ & $7.6,2.2$ & 2.76 & $\mathrm{dd}$ & 1.9 \\
\hline 22 & $1.53-1.25$ & $\mathrm{~m}(5 \mathrm{H})$ & & 1.38 & $\mathrm{~m}$ & \\
\hline 23 & $1.53-1.25$ & $\mathrm{~m}(5 \mathrm{H})$ & & $1.55-1.25$ & $\mathrm{~m}(4 \mathrm{H})$ & \\
\hline 24 & $1.53-1.25$ & $\mathrm{~m}(5 \mathrm{H})$ & & $1.55-1.25$ & $\mathrm{~m}(4 \mathrm{H})$ & \\
\hline $25(3 \mathrm{H})$ & 0.90 & $\mathrm{t}$ & 7.2 & 0.91 & $\mathrm{t}$ & 6.8 \\
\hline $26(3 \mathrm{H})$ & 2.26 & $\mathrm{~d}$ & 1.1 & 2.27 & $\mathrm{~s}$ & - \\
\hline $27 \mathrm{a}$ & 5.37 & $\mathrm{~s}$ & - & 5.36 & $\mathrm{~s}$ & - \\
\hline $27 \mathrm{~b}$ & 5.24 & $\mathrm{~s}$ & - & 5.21 & $\mathrm{~s}$ & - \\
\hline $28 \mathrm{a}$ & 5.51 & $\mathrm{~s}$ & - & 5.49 & $\mathrm{~s}$ & - \\
\hline $28 b$ & 5.43 & $\mathrm{~s}$ & - & 5.37 & $\mathrm{~s}$ & - \\
\hline $29 a$ & 4.84 & $\mathrm{~s}$ & - & 4.88 & $\mathrm{~s}$ & - \\
\hline $29 b$ & 4.76 & $\mathrm{~s}$ & - & 4.79 & $\mathrm{~s}$ & - \\
\hline $30(3 \mathrm{H})$ & 0.98 & $\mathrm{~d}$ & 6.6 & 1.06 & $\mathrm{~d}$ & 7.0 \\
\hline $31(3 \mathrm{H})$ & 0.97 & $\mathrm{~d}$ & 6.7 & 0.93 & $\mathrm{~d}$ & 6.0 \\
\hline
\end{tabular}


${ }^{1} \mathrm{H}$ NMR data for $\mathbf{5 7}$ in $\mathrm{CDCl}_{3}$.

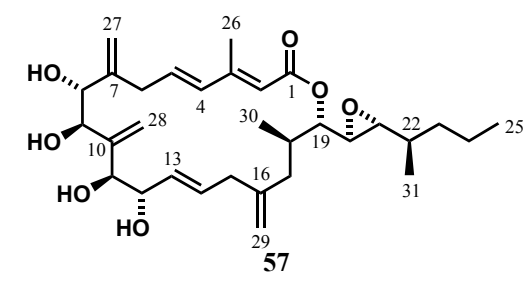

\begin{tabular}{|c|c|c|c|c|c|c|}
\hline & $\underline{\text { Isomer } 57}$ & & & Natural Product & & \\
\hline Proton \# & $\delta_{\mathrm{H}}$ & Mult. & $J$ & $\delta_{\mathrm{H}}$ & Mult. & $J$ \\
\hline 2 & 5.85 & $\mathrm{~s}$ & - & 5.80 & $\mathrm{~s}$ & - \\
\hline 4 & 6.27 & d & 15.5 & 6.27 & $\mathrm{~d}$ & 15.6 \\
\hline 5 & 6.08 & ddd & $15.1,10.0,5.0$ & 6.09 & ddd & $15.6,8.8,4.8$ \\
\hline $6 a$ & 3.20 & $\mathrm{dd}$ & $14.8,4.4$ & 3.20 & $\mathrm{dd}$ & $14.6,4.8$ \\
\hline $6 b$ & 3.10 & dd & $14.9,10.0$ & 3.13 & $\mathrm{dd}$ & $14.6,8.8$ \\
\hline 8 & 4.41 & $\mathrm{~s}$ br & & 4.42 & $\mathrm{~s}$ br & - \\
\hline 9 & 4.62 & $\mathrm{~s} b r$ & & 4.58 & $\mathrm{sbr}$ & - \\
\hline 11 & 4.04 & $\mathrm{~s} b r$ & & 4.09 & $\mathrm{~s} b r$ & - \\
\hline 12 & 4.20 & $\mathrm{~s}$ br & & 4.22 & $\mathrm{sbr}$ & - \\
\hline 13 & 5.49 & dd & $15.4,5.0$ & 5.51 & $\mathrm{dd}$ & $15.4,4.1$ \\
\hline 14 & 5.68 & dddd & $15.4,7.1,7.1,1.2$ & 5.69 & $\mathrm{dt}$ & $15.4,7.7,7.7$ \\
\hline $15 \mathrm{a}$ & $2.75-2.72$ & $\mathrm{~m}(2 \mathrm{H})$ & & 2.76 & $\mathrm{~s}$ br $(2 \mathrm{H})$ & \\
\hline $15 b$ & $2.75-2.72$ & $\mathrm{~m}(2 \mathrm{H})$ & & 2.76 & $\mathrm{~s}$ br $(2 \mathrm{H})$ & \\
\hline $17 \mathrm{a}$ & 2.51 & $\mathrm{dd}$ & $13.7,3.4$ & 2.35 & $\mathrm{dd}$ & $14.0,5.5$ \\
\hline $17 \mathrm{~b}$ & 1.85 & $\mathrm{dd}$ & $14.0,10.5$ & 1.92 & $\mathrm{dd}$ & $14.0,9.5$ \\
\hline 18 & 2.12 & $\mathrm{~m}$ & & 2.17 & $\mathrm{~m}$ & \\
\hline 19 & 4.65 & $\mathrm{dd}$ & $6.7,3.7$ & 4.72 & dd & $5.9,3.4$ \\
\hline 20 & 2.92 & $\mathrm{dd}$ & $6.7,2.2$ & 2.85 & $\mathrm{dd}$ & $5.9,1.9$ \\
\hline 21 & 2.68 & $\mathrm{dd}$ & $7.5,2.2$ & 2.76 & $\mathrm{dd}$ & 1.9 \\
\hline 22 & $1.53-1.24$ & $\mathrm{~m}(5 \mathrm{H})$ & & 1.38 & $\mathrm{~m}$ & \\
\hline 23 & $1.53-1.24$ & $\mathrm{~m}(5 \mathrm{H})$ & & $1.55-1.25$ & $\mathrm{~m}(4 \mathrm{H})$ & \\
\hline 24 & $1.53-1.24$ & $\mathrm{~m}(5 \mathrm{H})$ & & $1.55-1.25$ & $\mathrm{~m}(4 \mathrm{H})$ & \\
\hline $25(3 \mathrm{H})$ & 0.90 & $\mathrm{t}$ & 7.2 & 0.91 & $\mathrm{t}$ & 6.8 \\
\hline $26(3 \mathrm{H})$ & 2.27 & $\mathrm{~d}$ & 0.9 & 2.27 & $\mathrm{~s}$ & - \\
\hline $27 \mathrm{a}$ & 5.34 & $\mathrm{~s}$ & - & 5.36 & $\mathrm{~s}$ & - \\
\hline $27 b$ & 5.19 & $\mathrm{~s}$ & - & 5.21 & $\mathrm{~s}$ & - \\
\hline $28 \mathrm{a}$ & 5.45 & $\mathrm{~s}$ & - & 5.49 & $\mathrm{~s}$ & - \\
\hline $28 \mathrm{~b}$ & 5.34 & $\mathrm{~s}$ & - & 5.37 & $\mathrm{~s}$ & - \\
\hline $29 a$ & 4.85 & $\mathrm{~s}$ & - & 4.88 & $\mathrm{~s}$ & - \\
\hline $29 b$ & 4.77 & $\mathrm{~s}$ & - & 4.79 & $\mathrm{~s}$ & - \\
\hline $30(3 \mathrm{H})$ & 1.00 & d & 7.1 & 1.06 & $\mathrm{~d}$ & 7.0 \\
\hline $31(3 \mathrm{H})$ & 0.97 & d & 6.8 & 0.93 & $\mathrm{~d}$ & 6.0 \\
\hline
\end{tabular}


${ }^{1} \mathrm{H}$ NMR data for 59 in $\mathrm{CDCl}_{3}$.

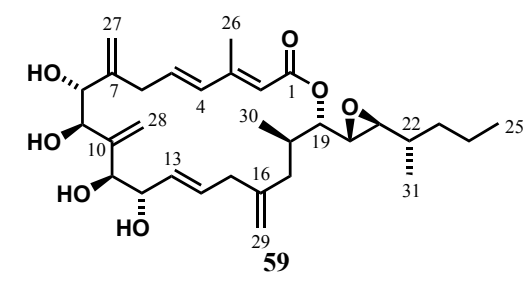

\begin{tabular}{|c|c|c|c|c|c|c|}
\hline & $\underline{\text { Isomer } 59}$ & & & Natural Product & & \\
\hline Proton \# & $\delta_{\mathrm{H}}$ & Mult. & $J$ & $\delta_{\mathrm{H}}$ & Mult. & $J$ \\
\hline 2 & 5.80 & $\mathrm{~s}$ & - & 5.80 & $\mathrm{~s}$ & - \\
\hline 4 & 6.28 & d & 15.4 & 6.27 & $\mathrm{~d}$ & 15.6 \\
\hline 5 & 6.10 & ddd & $15.5,9.6,5.1$ & 6.09 & ddd & $15.6,8.8,4.8$ \\
\hline $6 a$ & 3.21 & $\mathrm{dd}$ & $14.9,5.0$ & 3.20 & $\mathrm{dd}$ & $14.6,4.8$ \\
\hline $6 b$ & 3.11 & $\mathrm{dd}$ & $14.7,9.3$ & 3.13 & $\mathrm{dd}$ & $14.6,8.8$ \\
\hline 8 & 4.43 & $\mathrm{~s}$ br & - & 4.42 & $\mathrm{~s}$ br & - \\
\hline 9 & 4.59 & $\mathrm{~s} b r$ & - & 4.58 & $\mathrm{sbr}$ & - \\
\hline 11 & 4.06 & $\mathrm{tbr}$ & 3.8 & 4.09 & $\mathrm{~s} b r$ & - \\
\hline 12 & 4.22 & $\mathrm{~s} b r$ & - & 4.22 & $\mathrm{sbr}$ & - \\
\hline 13 & 5.50 & dd & $15.4,4.8$ & 5.51 & $\mathrm{dd}$ & $15.4,4.1$ \\
\hline 14 & 5.69 & dddd & $15.3,7.3,7.3,1.5$ & 5.69 & $\mathrm{dt}$ & $15.4,7.7,7.7$ \\
\hline $15 \mathrm{a}$ & 2.76 & $\mathrm{~s}$ br $(2 \mathrm{H})$ & & 2.76 & $\mathrm{~s}$ br $(2 \mathrm{H})$ & \\
\hline $15 b$ & 2.76 & $\mathrm{~s}$ br $(2 \mathrm{H})$ & & 2.76 & $\mathrm{~s}$ br $(2 \mathrm{H})$ & \\
\hline $17 \mathrm{a}$ & 2.34 & $\mathrm{dd}$ & $15.3,5.0$ & 2.35 & $\mathrm{dd}$ & $14.0,5.5$ \\
\hline $17 \mathrm{~b}$ & 1.92 & $\mathrm{dd}$ & $14.4,9.2$ & 1.92 & $\mathrm{dd}$ & $14.0,9.5$ \\
\hline 18 & 2.16 & $\mathrm{~m}$ & & 2.17 & $\mathrm{~m}$ & \\
\hline 19 & 4.72 & $\mathrm{dd}$ & $6.1,3.2$ & 4.72 & dd & $5.9,3.4$ \\
\hline 20 & 2.85 & $\mathrm{dd}$ & $6.2,2.2$ & 2.85 & $\mathrm{dd}$ & $5.9,1.9$ \\
\hline 21 & 2.76 & $\mathrm{dd}$ & $7.5,2.2$ & 2.76 & $\mathrm{dd}$ & 1.9 \\
\hline 22 & $1.53-1.24$ & $\mathrm{~m}(5 \mathrm{H})$ & & 1.38 & $\mathrm{~m}$ & \\
\hline 23 & $1.53-1.24$ & $\mathrm{~m}(5 \mathrm{H})$ & & $1.55-1.25$ & $\mathrm{~m}(4 \mathrm{H})$ & \\
\hline 24 & $1.53-1.24$ & $\mathrm{~m}(5 \mathrm{H})$ & & $1.55-1.25$ & $\mathrm{~m}(4 \mathrm{H})$ & \\
\hline $25(3 \mathrm{H})$ & 0.91 & $\mathrm{t}$ & 7.1 & 0.91 & $\mathrm{t}$ & 6.8 \\
\hline $26(3 \mathrm{H})$ & 2.27 & $\mathrm{~d}$ & 1.1 & 2.27 & $\mathrm{~s}$ & - \\
\hline $27 \mathrm{a}$ & 5.36 & d & 1.0 & 5.36 & $\mathrm{~s}$ & - \\
\hline $27 b$ & 5.21 & $\mathrm{~s}$ & - & 5.21 & $\mathrm{~s}$ & - \\
\hline $28 \mathrm{a}$ & 5.49 & $\mathrm{~s}$ & - & 5.49 & $\mathrm{~s}$ & - \\
\hline $28 \mathrm{~b}$ & 5.38 & d & 1.0 & 5.37 & $\mathrm{~s}$ & - \\
\hline $29 a$ & 4.88 & $\mathrm{~s}$ & - & 4.88 & $\mathrm{~s}$ & - \\
\hline $29 b$ & 4.79 & $\mathrm{~s}$ & - & 4.79 & $\mathrm{~s}$ & - \\
\hline $30(3 \mathrm{H})$ & 1.06 & d & 7.0 & 1.06 & $\mathrm{~d}$ & 7.0 \\
\hline $31(3 \mathrm{H})$ & 0.93 & d & 6.8 & 0.93 & $\mathrm{~d}$ & 6.0 \\
\hline
\end{tabular}


${ }^{1} \mathrm{H}$ NMR data for 59 in $\mathrm{C}_{6} \mathrm{D}_{6}$.

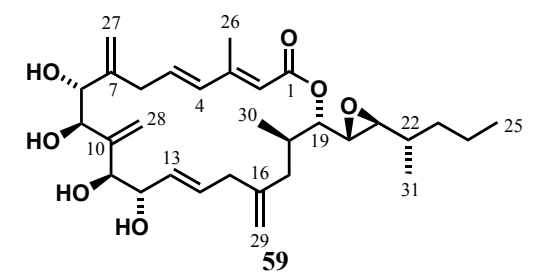

\begin{tabular}{|c|c|c|c|c|c|c|}
\hline & $\underline{\text { Isomer } 59}$ & & & Natural Product & & \\
\hline Proton \# & $\delta_{\mathrm{H}}$ & Mult. & $J$ & $\delta_{\mathrm{H}}$ & Mult. & $J$ \\
\hline 2 & 5.83 & $\mathrm{~s}$ & - & 5.83 & $\mathrm{~s}$ & - \\
\hline 4 & 5.98 & $\mathrm{~d}$ & 15.6 & 5.98 & $\mathrm{~d}$ & 15.6 \\
\hline 5 & 5.71 & ddd & $14.9,8.9,5.6$ & 5.71 & ddd & $15.6,8.9,5.7$ \\
\hline $6 a$ & 2.88 & $\mathrm{~m}$ & & 2.87 & $\mathrm{~m}$ & \\
\hline $6 \mathrm{~b}$ & 2.88 & $\mathrm{~m}$ & & 2.87 & $\mathrm{~m}$ & \\
\hline 8 & 4.39 & $\mathrm{~s} b r$ & - & 4.38 & $\mathrm{~s} b r$ & - \\
\hline 9 & 4.45 & $\mathrm{~s} b r$ & - & 4.44 & $\mathrm{~s}$ br & - \\
\hline 11 & 3.90 & $\mathrm{t} b r$ & 3.9 & 3.89 & $\mathrm{~s} b r$ & - \\
\hline 12 & 3.97 & $\mathrm{~s} \mathrm{br}$ & - & 3.98 & $\mathrm{sbr}$ & - \\
\hline 13 & 5.29 & $\mathrm{dd}$ & $15.3,4.4$ & 5.29 & $\mathrm{dd}$ & $15.4,4.4$ \\
\hline 14 & 5.65 & ddd & $13.7,7.4,5.7$ & 5.65 & ddd & $15.4,7.4,6.5$ \\
\hline $15 \mathrm{a}$ & 2.63 & $\mathrm{dd}$ & $14.0,7.1$ & 2.63 & $\mathrm{dd}$ & $14.4,7.4$ \\
\hline $15 b$ & 2.56 & $\mathrm{dd}$ & $14.2,7.1$ & 2.56 & $\mathrm{dd}$ & $14.4,6.5$ \\
\hline $17 \mathrm{a}$ & 2.36 & $\mathrm{dd}$ & $14.5,4.4$ & 2.36 & $\mathrm{dd}$ & $14.7,6.2$ \\
\hline $17 b$ & 1.87 & $\mathrm{dd}$ & $14.4,8.8$ & 1.88 & $\mathrm{dd}$ & $14.7,8.9$ \\
\hline 18 & 2.19 & $\mathrm{~m}$ & & 2.19 & $\mathrm{~m}$ & \\
\hline 19 & 4.92 & $\mathrm{dd}$ & $6.8,3.1$ & 4.92 & $\mathrm{dd}$ & $6.8,3.1$ \\
\hline 20 & 2.79 & $\mathrm{dd}$ & $6.7,2.1$ & 2.79 & $\mathrm{dd}$ & $6.8,2.0$ \\
\hline 21 & 2.85 & $\mathrm{dd}$ & $7.6,2.0$ & 2.85 & $\mathrm{dd}$ & $7.5,2.0$ \\
\hline 22 & $1.48-1.12$ & $\mathrm{~m}(5 \mathrm{H})$ & & 1.24 & $\mathrm{~m}$ & \\
\hline 23 & $1.48-1.12$ & $\mathrm{~m}(5 \mathrm{H})$ & & $1.49-1.13$ & $\mathrm{~m}(4 \mathrm{H})$ & \\
\hline 24 & $1.48-1.12$ & $\mathrm{~m}(5 \mathrm{H})$ & & $1.49-1.13$ & $\mathrm{~m}(4 \mathrm{H})$ & \\
\hline $25(3 \mathrm{H})$ & 0.85 & $\mathrm{t}$ & 7.2 & 0.85 & $\mathrm{t}$ & 7.3 \\
\hline $26(3 \mathrm{H})$ & 2.21 & $d$ & 1.1 & 2.21 & $\mathrm{~s}$ & - \\
\hline $27 \mathrm{a}$ & 5.46 & $\mathrm{~s}$ & - & 5.45 & $\mathrm{~s}$ & - \\
\hline $27 b$ & 5.01 & $\mathrm{~s} b r$ & - & 5.01 & $\mathrm{~s} b r$ & - \\
\hline $28 \mathrm{a}$ & 5.43 & $\mathrm{t}$ & 1.3 & 5.42 & $\mathrm{t}$ & 1.3 \\
\hline $28 \mathrm{~b}$ & 5.15 & d & 1.1 & 5.15 & $\mathrm{~d}$ & 1.3 \\
\hline $29 a$ & 4.86 & $\mathrm{~s}$ & - & 4.86 & $\mathrm{~s}$ & - \\
\hline $29 b$ & 4.78 & $\mathrm{~d}$ & 1.2 & 4.78 & $\mathrm{~d}$ & 1.5 \\
\hline $30(3 \mathrm{H})$ & 1.09 & $d$ & 7.0 & 1.09 & $\mathrm{~d}$ & 7.0 \\
\hline $31(3 \mathrm{H})$ & 0.83 & $\mathrm{~d}$ & 6.8 & 0.83 & $\mathrm{~d}$ & 6.8 \\
\hline
\end{tabular}


${ }^{1} \mathrm{H}$ NMR data for $\mathbf{5 9}$ in $\mathrm{CD}_{3} \mathrm{OD}$.

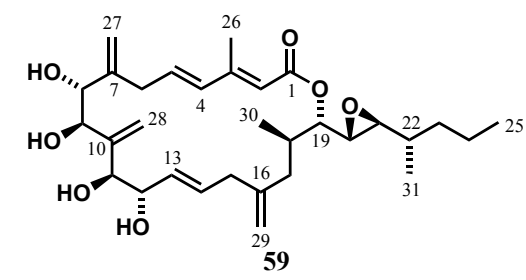

\begin{tabular}{|c|c|c|c|c|c|c|}
\hline \multirow[b]{2}{*}{ Proton \# } & \multicolumn{3}{|l|}{$\underline{\text { Isomer } 59}$} & \multicolumn{3}{|c|}{$\underline{\text { Natural Product }}$} \\
\hline & $\delta_{\mathrm{H}}$ & Mult. & $J$ & $\delta_{\mathrm{H}}$ & Mult. & $J$ \\
\hline 2 & 5.85 & $\mathrm{~s}$ & - & 5.85 & $\mathrm{~s}$ & - \\
\hline 4 & 6.36 & $\mathrm{~d}$ & 15.4 & 6.35 & $\mathrm{~d}$ & 15.4 \\
\hline 5 & 6.14 & ddd & $15.1,8.9,5.9$ & 6.14 & ddd & $15.4,9.0,5.9$ \\
\hline $6 a$ & $3.12-3.09$ & $\mathrm{~m}(2 \mathrm{H})$ & & 3.10 & $\mathrm{~m}$ & \\
\hline $6 \mathrm{~b}$ & $3.12-3.09$ & $\mathrm{~m}(2 \mathrm{H})$ & & 3.10 & $\mathrm{~m}$ & \\
\hline 8 & 4.21 & $\mathrm{~s}$ br & - & 4.21 & $\mathrm{~s}$ br & - \\
\hline 9 & 4.46 & $\mathrm{~s}$ br & - & 4.46 & $\mathrm{~s}$ br & - \\
\hline 11 & 3.99 & $\mathrm{~d} b r$ & 2.2 & 3.99 & $\mathrm{~d}$ & 2.8 \\
\hline 12 & 4.13 & $\mathrm{~s}$ br & - & 4.14 & $\mathrm{~s}$ br & - \\
\hline 13 & 5.46 & $\mathrm{dd}$ & $15.3,5.3$ & 5.46 & $\mathrm{dd}$ & $15.6,5.5$ \\
\hline 14 & 5.59 & dddd & $15.3,7.7,6.2,1.5$ & 5.59 & ddd & $15.6,8.4,5.8$ \\
\hline $15 \mathrm{a}$ & 2.67 & dd & $14.5,7.9$ & 2.67 & $\mathrm{dd}$ & $14.3,8.4$ \\
\hline $15 b$ & 2.75 & dd & $14.3,6.2$ & 2.75 & dd & $14.3,5.8$ \\
\hline $17 \mathrm{a}$ & 2.41 & $\mathrm{dd}$ & $13.9,5.1$ & 2.41 & $\mathrm{dd}$ & $14.3,5.8$ \\
\hline $17 \mathrm{~b}$ & 1.92 & $\mathrm{dd}$ & $13.7,8.8$ & 1.92 & $\mathrm{dd}$ & $14.7,8.5$ \\
\hline 18 & 2.14 & $\mathrm{~m}$ & & 2.14 & $\mathrm{~m}$ & \\
\hline 19 & 4.69 & dd & $5.7,3.3$ & 4.69 & dd & $5.7,3.2$ \\
\hline 20 & 2.91 & dd & $5.7,2.2$ & 2.91 & dd & $5.7,2.2$ \\
\hline 21 & 2.75 & dd & $7.6,2.2$ & 2.75 & $\mathrm{dd}$ & $7.4,2.2$ \\
\hline 22 & $1.50-1.25$ & $\mathrm{~m}(5 \mathrm{H})$ & & 1.37 & $\mathrm{~m}$ & \\
\hline 23 & $1.50-1.25$ & $\mathrm{~m}(5 \mathrm{H})$ & & $1.50-1.25$ & $\mathrm{~m}(4 \mathrm{H})$ & \\
\hline 24 & $1.50-1.25$ & $\mathrm{~m}(5 \mathrm{H})$ & & $1.50-1.25$ & $\mathrm{~m}(4 \mathrm{H})$ & \\
\hline $25(3 \mathrm{H})$ & 0.91 & $\mathrm{t}$ & 7.1 & 0.91 & $\mathrm{t}$ & 7.2 \\
\hline $26(3 \mathrm{H})$ & 2.25 & $\mathrm{~d}$ & 1.2 & 2.25 & $\mathrm{~s}$ & - \\
\hline $27 \mathrm{a}$ & 5.27 & $\mathrm{~s}$ & 1.6 & 5.27 & $\mathrm{t}$ & 1.2 \\
\hline $27 b$ & 5.10 & $\mathrm{~s}$ br & - & 5.10 & $\mathrm{~s}$ br & - \\
\hline $28 \mathrm{a}$ & 5.40 & $\mathrm{t}$ & 1.5 & 5.40 & $\mathrm{t}$ & 1.3 \\
\hline $28 b$ & 5.34 & $\mathrm{~s}$ & - & 5.35 & $\mathrm{~s} \mathrm{br}$ & - \\
\hline $29 a$ & 4.85 & $\mathrm{~s}$ & - & 4.85 & $\mathrm{~s}$ & - \\
\hline $29 b$ & 4.76 & $\mathrm{~s}$ br & - & 4.76 & $\mathrm{~s}$ br & - \\
\hline $30(3 \mathrm{H})$ & 1.06 & $\mathrm{~d}$ & 7.1 & 1.06 & $\mathrm{~d}$ & 7.1 \\
\hline $31(3 \mathrm{H})$ & 0.92 & $\mathrm{~d}$ & 6.7 & 0.92 & $\mathrm{~d}$ & 6.8 \\
\hline
\end{tabular}


${ }^{13} \mathrm{C}$ NMR data for $\mathbf{5 9}$ in $\mathrm{CDCl}_{3}$.

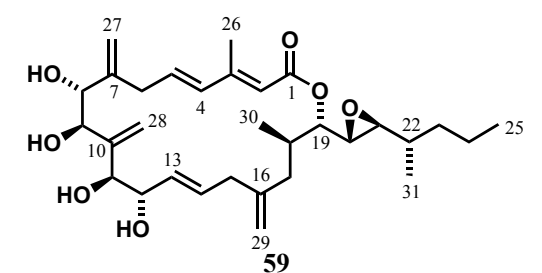

\begin{tabular}{|c|c|c|}
\hline & Isomer $\mathbf{5 9}$ & $\underline{\text { Natural Product }}$ \\
\hline Carbon \# & $\delta_{\mathrm{C}}$ & $\delta_{\mathrm{C}}$ \\
\hline 1 & 165.8 & 165.8 \\
\hline 2 & 118.6 & 118.6 \\
\hline 3 & 152.7 & 152.8 \\
\hline 4 & 136.3 & 136.4 \\
\hline 5 & 134.7 & 134.8 \\
\hline 6 & 39.0 & 39.0 \\
\hline 7 & 144.8 & 144.8 \\
\hline 8 & 72.5 & 72.5 \\
\hline 9 & 70.6 & 70.5 \\
\hline 10 & 147.2 & 147.2 \\
\hline 11 & 75.8 & 75.8 \\
\hline 12 & 73.5 & 73.5 \\
\hline 13 & 130.5 & 130.6 \\
\hline 14 & 130.9 & 130.9 \\
\hline 15 & 39.7 & 39.7 \\
\hline 16 & 145.0 & 145.0 \\
\hline 17 & 36.2 & 36.2 \\
\hline 18 & 33.3 & 33.3 \\
\hline 19 & 74.7 & 74.7 \\
\hline 20 & 54.2 & 54.2 \\
\hline 21 & 61.8 & 61.8 \\
\hline 22 & 35.3 & 35.4 \\
\hline 23 & 36.7 & 36.7 \\
\hline 24 & 19.9 & 20.0 \\
\hline 25 & 14.2 & 14.3 \\
\hline 26 & 13.9 & 13.9 \\
\hline 27 & 114.6 & 114.7 \\
\hline 28 & 116.0 & 116.1 \\
\hline 29 & 112.8 & 112.9 \\
\hline 30 & 14.9 & 14.9 \\
\hline 31 & 15.8 & 15.8 \\
\hline
\end{tabular}




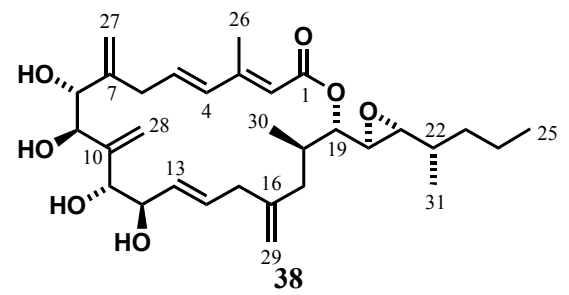

$\mathrm{R}_{f}=0.31$ (EtOAc); ${ }^{1} \mathrm{H}$ NMR $\left(500 \mathrm{MHz}, \mathrm{CDCl}_{3}\right) \delta 6.19(\mathrm{~d}, J=15.6 \mathrm{~Hz}, 1 \mathrm{H}), 6.08$ (ddd, $J=14.9$, 9.8, 3.8 Hz, 1H), $5.79(\mathrm{~s}, 1 \mathrm{H}), 5.77-5.72(\mathrm{~m}, 1 \mathrm{H}), 5.59(\mathrm{~s}, 1 \mathrm{H}), 5.57(\mathrm{~s}, 1 \mathrm{H}), 5.37(\mathrm{~s}, 1 \mathrm{H}), 5.26(\mathrm{~d}$ br, $J=16.2 \mathrm{~Hz}, 1 \mathrm{H}), 5.22(\mathrm{~s}, 1 \mathrm{H}), 4.86(\mathrm{~s}, 1 \mathrm{H}), 4.78(\mathrm{~s}, 1 \mathrm{H}), 4.61(\mathrm{dd}, J=6.2,3.8 \mathrm{~Hz}, 1 \mathrm{H}), 4.28$ (s, 1H), 4.23 (s, 1H), 4.20 (s, 1H), 3.83 (s, 1H), 3.22 (dd, $J=15.1,4.4 \mathrm{~Hz}, 1 \mathrm{H}), 3.11$ (dd, $J=$ 15.3, 10.4 Hz, 1H), 2.97 (dd, $J=6.1,2.1 \mathrm{~Hz}, 1 \mathrm{H}), 2.76$ (dd, $J=13.6,4.0 \mathrm{~Hz}, 1 \mathrm{H}), 2.69$ (dd, $J=$ 8.9, $2.1 \mathrm{~Hz}, 1 \mathrm{H}), 2.66$ (dd, $J=14.2,10.4 \mathrm{~Hz}, 1 \mathrm{H}), 2.51$ (dd, $J=12.5,2.5 \mathrm{~Hz}, 1 \mathrm{H}), 2.29$ (s, 3H), $2.13(\mathrm{~m}, 1 \mathrm{H}), 1.79(\mathrm{dd}, J=12.7,11.6 \mathrm{~Hz}, 1 \mathrm{H}), 1.44-1.22(\mathrm{~m}, 5 \mathrm{H}), 1.01$ (d, $J=6.4 \mathrm{~Hz}, 3 \mathrm{H}), 0.99$ $(\mathrm{d}, J=7.0 \mathrm{~Hz}, 3 \mathrm{H}), 0.91$ (t, $J=7.0 \mathrm{~Hz}, 3 \mathrm{H})$, exchangeable protons not listed; ${ }^{13} \mathrm{C}$ NMR (126 $\left.\mathrm{MHz}, \mathrm{CDCl}_{3}\right) \delta$ 165.6, 152.1, 146.6, 145.2, 143.7, 136.3, 133.8, 131.2, 130.2, 119.3, 115.4, 114.8, 110.6, 78.8, 76.4, 72.5, 69.6, 65.9, 61.0, 52.6, 41.3, 38.6, 36.4, 35.3, 35.2, 33.4, 20.2, 15.9, 15.3, 14.2, 13.6; IR (neat) 3293 (br), 1709, 1234, $1152 \mathrm{~cm}^{-1}$; ESIMS calcd for $\mathrm{C}_{31} \mathrm{H}_{46} \mathrm{O}_{7} \mathrm{Na}\left(\mathrm{M}^{+}+\right.$ $\mathrm{Na}) 553.3$, found 553.3 ; optical rotation $[\alpha]_{\mathrm{D}}^{23}+55^{\circ}\left(c 0.10, \mathrm{CH}_{2} \mathrm{Cl}_{2}\right)$.

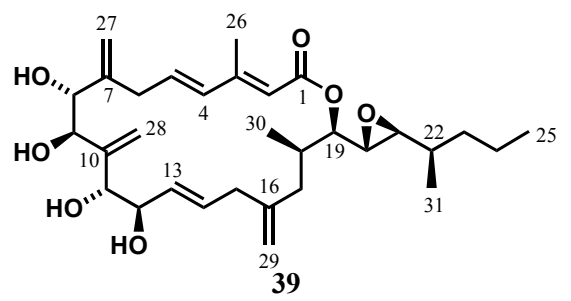

$\mathrm{R}_{f}=0.38$ (EtOAc); ${ }^{1} \mathrm{H}$ NMR $\left(500 \mathrm{MHz}, \mathrm{CDCl}_{3}\right) \delta 6.19(\mathrm{~d}, J=15.4 \mathrm{~Hz}, 1 \mathrm{H}), 6.08$ (ddd, $J=14.7$, 8.1, $6.4 \mathrm{~Hz}, 1 \mathrm{H}), 5.81(\mathrm{~s}, 1 \mathrm{H}), 5.72$ (dddd, $J=15.4,8.6,7.0,1.2 \mathrm{~Hz}, 1 \mathrm{H}), 5.49$ (s, $1 \mathrm{H}), 5.48$ (ddm, $J=15.6,4.9 \mathrm{~Hz}, 1 \mathrm{H}), 5.44$ (s, 1H), 5.34 (s, 1H), 5.24 (s, 1H), 4.83 (s, 1H), 4.75 (s, 1H), $4.60(\mathrm{dd}, J=10.3,5.5 \mathrm{~Hz}, 1 \mathrm{H}), 4.32$ (s, 1H), 4.16 (s, 1H), 4.09 (m, 1H), 3.95 (s, 1H), 3.25 (dd, $J$ $=14.4,6.4 \mathrm{~Hz}, 1 \mathrm{H}), 3.02(\mathrm{dd}, J=5.6,2.2 \mathrm{~Hz}, 1 \mathrm{H}), 3.00(\mathrm{dd}, J=14.4,8.2 \mathrm{~Hz}, 1 \mathrm{H}), 2.76-2.74(\mathrm{~m}$, 2H), $2.72(\mathrm{dd}, J=6.7,2.1 \mathrm{~Hz}, 1 \mathrm{H}), 2.26(\mathrm{~d}, J=1.0 \mathrm{~Hz}, 3 \mathrm{H}), 2.17(\mathrm{dd}, J=12.9,2.6 \mathrm{~Hz}, 1 \mathrm{H}), 1.94$ $(\mathrm{m}, 1 \mathrm{H}), 1.65(\mathrm{dd}, J=13.3,9.6 \mathrm{~Hz}, 1 \mathrm{H}), 1.47-1.21(\mathrm{~m}, 5 \mathrm{H}), 1.04(\mathrm{~d}, J=6.7 \mathrm{~Hz}, 3 \mathrm{H}), 1.00(\mathrm{~d}, J=$ $6.6 \mathrm{~Hz}, 3 \mathrm{H}), 0.92(\mathrm{t}, J=7.2 \mathrm{~Hz}, 3 \mathrm{H})$, exchangeable protons not listed; ${ }^{13} \mathrm{C} \mathrm{NMR}(101 \mathrm{MHz}$, 
$\left.\mathrm{CDCl}_{3}\right) \delta 165.9,152.1,146.9,145.0,144.9,135.6,134.7,131.7,130.2,118.5,115.6,115.0$, $113.5,77.2,76.1,74.8,74.3,72.5,72.3,62.4,58.4,39.5,39.2,38.8,35.4,35.0,33.6,20.0,16.4$, 15.7, 14.2; IR (neat) 3286 (br), 2963, 1706, 1616, $1150 \mathrm{~cm}^{-1}$; ESIMS calcd for $\mathrm{C}_{31} \mathrm{H}_{46} \mathrm{O}_{7} \mathrm{Na}\left(\mathrm{M}^{+}\right.$ $+\mathrm{Na}) 553.3$, found 553.3; optical rotation $[\alpha]_{\mathrm{D}}^{23}-65^{\circ}\left(c 0.10, \mathrm{CH}_{2} \mathrm{Cl}_{2}\right)$.

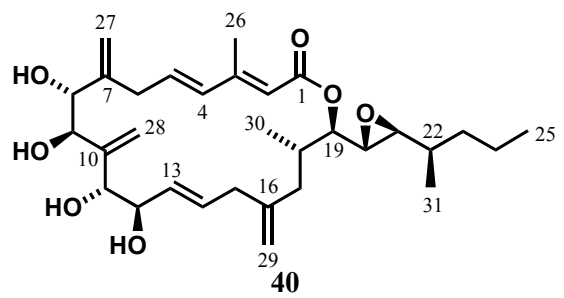

$\mathrm{R}_{f}=0.30$ (EtOAc); ${ }^{1} \mathrm{H}$ NMR $\left(500 \mathrm{MHz}, \mathrm{CDCl}_{3}\right) \delta 6.16(\mathrm{~d}, J=15.9 \mathrm{~Hz}, 1 \mathrm{H}), 6.08$ (ddd, $J=15.5$, 7.0, $6.7 \mathrm{~Hz}, 1 \mathrm{H}), 5.82$ (s, 1H), 5.68 (ddd, $J=15.4,7.8,6.6,1.2 \mathrm{~Hz}, 1 \mathrm{H}), 5.47$ (s, 1H), 5.39 (s, 1H), 5.38 (dd, $J=15.4,5.4 \mathrm{~Hz}, 1 \mathrm{H}), 5.32(\mathrm{~s}, 1 \mathrm{H}), 5.20(\mathrm{~s}, 1 \mathrm{H}), 4.85$ (s br, 1H), 4.77 (s br, 1H), 4.67 (dd, $J=6.1,3.4 \mathrm{~Hz}, 1 \mathrm{H}), 4.38(\mathrm{~d}, J=4.3 \mathrm{~Hz}, 1 \mathrm{H}), 4.17$ (dd, $J=4.0,3.7 \mathrm{~Hz}, 1 \mathrm{H}), 4.10$ (d, $J$ $=4.3 \mathrm{~Hz}, 1 \mathrm{H}), 3.89(\mathrm{~d}, J=3.5 \mathrm{~Hz}, 1 \mathrm{H}), 3.21(\mathrm{dd}, J=14.7,6.5 \mathrm{~Hz}, 1 \mathrm{H}), 2.98(\mathrm{dd}, J=14.5,7.4$ $\mathrm{Hz}, 1 \mathrm{H}), 2.97$ (dd, $J=6.1,2.2 \mathrm{~Hz}, 1 \mathrm{H}), 2.73(\mathrm{dd}, J=13.9,6.0 \mathrm{~Hz}, 1 \mathrm{H}), 2.66(\mathrm{dd}, J=7.0,2.2 \mathrm{~Hz}$, 1H), $2.63(\mathrm{dd}, J=14.7,8.3 \mathrm{~Hz}, 1 \mathrm{H}), 2.40(\mathrm{dd}, J=13.7,3.9 \mathrm{~Hz}, 1 \mathrm{H}), 2.25(\mathrm{~d}, J=1.1 \mathrm{~Hz}, 3 \mathrm{H})$, $2.11(\mathrm{~m}, 1 \mathrm{H}), 1.82(\mathrm{dd}, J=13.7,10.1 \mathrm{~Hz}, 1 \mathrm{H}), 1.43-1.20(\mathrm{~m}, 5 \mathrm{H}), 1.01(\mathrm{~d}, J=7.0 \mathrm{~Hz}, 3 \mathrm{H}), 1.00$ (d, $J=6.5 \mathrm{~Hz}, 3 \mathrm{H}), 0.91(\mathrm{t}, J=7.0 \mathrm{~Hz}, 3 \mathrm{H})$, exchangeable protons not listed; ${ }^{13} \mathrm{C}$ NMR (125 $\left.\mathrm{MHz}, \mathrm{CDCl}_{3}\right) \delta$ 166.0, 152.4, 147.0, 145.1, 144.7, 133.4, 134.9, 131.5, 130.3, 118.3, 116.2, 115.0, 113.4, 76.4, 75.1, 74.1, 73.6, 72.2, 60.9, 55.8, 39.2, 38.3, 36.4, 35.4, 35.2, 34.1, 20.2, 16.7, 15.7, 14.4, 14.2; IR (neat) 3285, 2922, 1709, 1614, $1153 \mathrm{~cm}^{-1}$; ESIMS calcd for $\mathrm{C}_{31} \mathrm{H}_{46} \mathrm{O}_{7} \mathrm{Na}\left(\mathrm{M}^{+}\right.$ $+\mathrm{Na}) 553.3$, found 553.4 ; optical rotation $[\alpha]_{\mathrm{D}}^{26}-42^{\circ}\left(c 0.10, \mathrm{CH}_{2} \mathrm{Cl}_{2}\right)$.

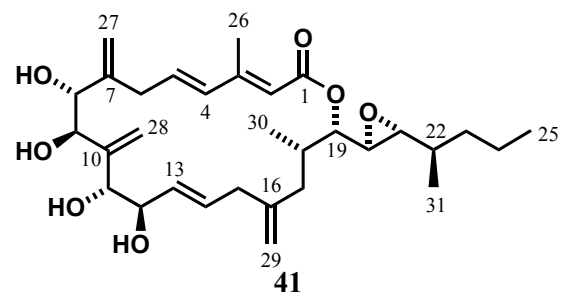

$\mathrm{R}_{f}=0.38\left(\right.$ EtOAc); ${ }^{1} \mathrm{H}$ NMR $\left(500 \mathrm{MHz}, \mathrm{CDCl}_{3}\right) \delta 6.16(\mathrm{~d}, J=15.6 \mathrm{~Hz}, 1 \mathrm{H}), 6.03(\mathrm{ddd}, J=14.9$, 9.8, $4.8 \mathrm{~Hz}, 1 \mathrm{H}), 5.75$ (ddd, $J=15.3,7.3,6.4 \mathrm{~Hz}, 1 \mathrm{H}), 5.72$ (s, 1H), 5.53 (dd, $J=15.6,5.4 \mathrm{~Hz}$, $1 \mathrm{H}), 5.49(\mathrm{~s}, 1 \mathrm{H}), 5.42(\mathrm{~s}, 1 \mathrm{H}), 5.33(\mathrm{~s}, 1 \mathrm{H}), 5.20(\mathrm{~s}, 1 \mathrm{H}), 4.74(\mathrm{~s}, 1 \mathrm{H}), 4.73(\mathrm{~s}, 1 \mathrm{H}), 4.56(\mathrm{dd}, J=$ 
10.3, 5.6 Hz, 1H), $4.36(\mathrm{~s} \mathrm{br}, 1 \mathrm{H}), 4.30(\mathrm{~s} \mathrm{br}, 1 \mathrm{H}), 4.27$ (s br, 1H), 3.99 (s br, $1 \mathrm{H}), 3.16$ (dd, $J=$ 14.4, $4.3 \mathrm{~Hz}, 1 \mathrm{H}), 3.04$ (dd, $J=14.7,9.9 \mathrm{~Hz}, 1 \mathrm{H}), 2.91(\mathrm{dd}, J=5.7,2.1 \mathrm{~Hz}, 1 \mathrm{H}), 2.81$ (dd, $J=$ $15.3,7.0 \mathrm{~Hz}, 1 \mathrm{H}), 2.70(\mathrm{dd}, J=7.6,2.0 \mathrm{~Hz}, 1 \mathrm{H}), 2.68(\mathrm{~m}, 1 \mathrm{H}), 2.24$ (s, 3H), 2.15 (dd, $J=13.8$, $4.8 \mathrm{~Hz}, 1 \mathrm{H}), 2.01(\mathrm{~m}, 1 \mathrm{H}), 1.76(\mathrm{dd}, J=13.9,8.4 \mathrm{~Hz}, 1 \mathrm{H}), 1.51-1.19(\mathrm{~m}, 5 \mathrm{H}), 1.02$ (d, $J=6.7$ $\mathrm{Hz}, 3 \mathrm{H}), 0.93(\mathrm{~d}, J=6.8 \mathrm{~Hz}, 3 \mathrm{H}), 0.89(\mathrm{t}, J=7.2 \mathrm{~Hz}, 3 \mathrm{H})$, exchangeable protons not listed; ${ }^{13} \mathrm{C}$ NMR (125 MHz, $\left.\mathrm{CDCl}_{3}\right) \delta 165.9,152.9,147.1,145.0,144.7,136.0,134.7,131.5,130.4,118.3$, 115.1, 114.7, 112.9, 76.1, 74.9, 73.5, 72.5, 70.8, 62.5, 57.8, 39.9, 38.4, 38.2, 36.6, 35.4, 33.3, 20.0, 16.2, 15.8, 14.2, 13.9; IR (neat) 3274 (br), 2829, 1719, 1610, 1231, $1144 \mathrm{~cm}^{-1}$; ESIMS calcd for $\mathrm{C}_{31} \mathrm{H}_{46} \mathrm{O}_{7} \mathrm{Na}\left(\mathrm{M}^{+}+\mathrm{Na}\right) 553.3$, found 553.4; optical rotation $[\alpha]_{\mathrm{D}}{ }^{26}+165^{\circ}(c 0.050$, $\mathrm{CH}_{2} \mathrm{Cl}_{2}$ ).

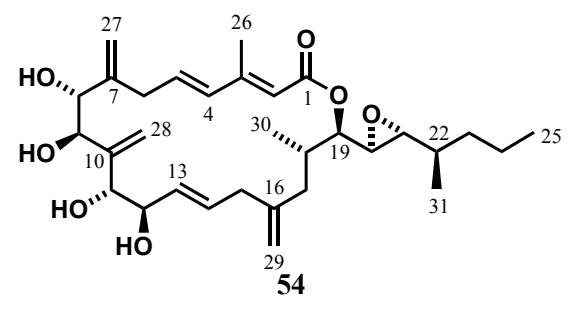

$\mathrm{R}_{f}=0.41\left(10 \% \mathrm{MeOH}\right.$ in $\left.\mathrm{CH}_{2} \mathrm{Cl}_{2}\right) ;{ }^{1} \mathrm{H}$ NMR $\left(500 \mathrm{MHz}, \mathrm{CDCl}_{3}\right) \delta 6.16(\mathrm{~d}, J=15.5 \mathrm{~Hz}, 1 \mathrm{H}), 6.08$ (ddd, $J=15.9,6.8,6.5 \mathrm{~Hz}, 1 \mathrm{H}$ ), 5.77 (s, 1H), 5.69 (ddd, $J=14.2,6.3,6.3 \mathrm{~Hz}, 1 \mathrm{H}$ ), 5.48 (s, $1 \mathrm{H}$ ), $5.41(\mathrm{dd}, J=15.5,5.4 \mathrm{~Hz}, 1 \mathrm{H}), 5.39$ (s, 1H), 5.33 (s, 1H), $5.21(\mathrm{~s}, 1 \mathrm{H}), 4.87(\mathrm{~s}, 1 \mathrm{H}), 4.79(\mathrm{~s}, 1 \mathrm{H})$, 4.67 (dd, $J=6.4,3.2 \mathrm{~Hz}, 1 \mathrm{H}), 4.38$ (s br, 1H), 4.18 (s br, 1H), 4.12 (s br, 1H), 3.91 (s br, 1H), $3.21(\mathrm{dd}, J=14.9,7.0 \mathrm{~Hz}, 1 \mathrm{H}), 2.98(\mathrm{dd}, J=14.4,7.1 \mathrm{~Hz}, 1 \mathrm{H}), 2.84(\mathrm{dd}, J=6.2,2.1 \mathrm{~Hz}, 1 \mathrm{H})$, $2.77(\mathrm{dd}, J=7.6,2.2 \mathrm{~Hz}, 1 \mathrm{H}), 2.74(\mathrm{dd}, J=14.8,6.7 \mathrm{~Hz}, 1 \mathrm{H}), 2.66(\mathrm{dd}, J=14.8,7.3 \mathrm{~Hz}, 1 \mathrm{H})$, $2.26(\mathrm{~d} \mathrm{br}, J=14.0 \mathrm{~Hz}, 1 \mathrm{H}), 2.24(\mathrm{~d}, J=1.0 \mathrm{~Hz}, 3 \mathrm{H}), 2.14(\mathrm{~m}, 1 \mathrm{H}), 1.88(\mathrm{dd}, J=13.9,9.2 \mathrm{~Hz}$, 1H), 1.51-1.25 (m, 5H), 1.05 (d, $J=7.1 \mathrm{~Hz}, 3 \mathrm{H}), 0.91$ (t, $J=7.0 \mathrm{~Hz}, 3 \mathrm{H}), 0.89$ (d, $J=7.1 \mathrm{~Hz}$, $3 \mathrm{H})$; IR (neat) 3368 (br), 2920, 1712, 1610, $1147 \mathrm{~cm}^{-1}$; ESIMS calcd for $\mathrm{C}_{31} \mathrm{H}_{46} \mathrm{O}_{7} \mathrm{Na}\left(\mathrm{M}^{+}+\mathrm{Na}\right)$ 553.3, found 553.3.

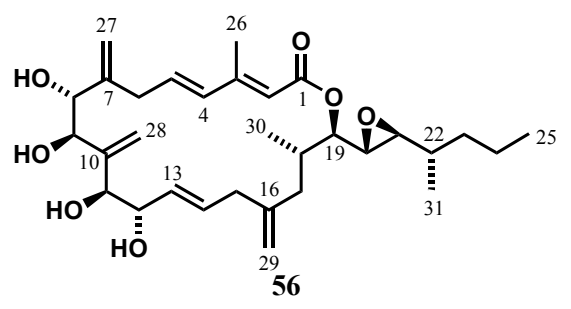


$\mathrm{R}_{f}=0.37$ (EtOAc); ${ }^{1} \mathrm{H}$ NMR $\left(500 \mathrm{MHz}, \mathrm{CDCl}_{3}\right) \delta 6.20(\mathrm{~d}, J=15.8 \mathrm{~Hz}, 1 \mathrm{H}), 6.08$ (ddd, $J=14.5$, 8.4, 5.6 Hz, 1H), 5.79 (s, 1H), 5.68 (dddd, $J=15.0,8.3,7.1,1.3 \mathrm{~Hz}, 1 \mathrm{H}), 5.51$ (s, 1H), 5.43 (s, 1H), 5.42 (dd, $J=15.4,5.4 \mathrm{~Hz}, 1 \mathrm{H}), 5.37$ (s, 1H), $5.24(\mathrm{~s}, 1 \mathrm{H}), 4.84(\mathrm{~s}, 1 \mathrm{H}), 4.76(\mathrm{~s}, 1 \mathrm{H}), 4.62$ $(\mathrm{dd}, J=6.7,3.5 \mathrm{~Hz}, 1 \mathrm{H}), 4.42(\mathrm{~d}, J=3.2 \mathrm{~Hz}, 1 \mathrm{H}), 4.28(\mathrm{~d}, J=3.2 \mathrm{~Hz}, 1 \mathrm{H}), 4.17(\mathrm{t}, J=4.5 \mathrm{~Hz}$, 1H), $4.02(\mathrm{~d}, J=3.9 \mathrm{~Hz}, 1 \mathrm{H}), 3.22(\mathrm{dd}, J=14.5,5.4 \mathrm{~Hz}, 1 \mathrm{H}), 2.94(\mathrm{dd}, J=13.1,8.9 \mathrm{~Hz}, 1 \mathrm{H})$, $2.92(\mathrm{dd}, J=7.0,2.3 \mathrm{~Hz}, 1 \mathrm{H}), 2.74(\mathrm{dd}, J=14.6,5.1 \mathrm{~Hz}, 1 \mathrm{H}), 2.68(\mathrm{dd}, J=7.6,2.2 \mathrm{~Hz}, 1 \mathrm{H})$, $2.67(\mathrm{dd}, J=14.4,7.7 \mathrm{~Hz}, 1 \mathrm{H}), 2.50(\mathrm{dd}, J=13.3,3.4 \mathrm{~Hz}, 1 \mathrm{H}), 2.26$ (d, $J=1.1 \mathrm{~Hz}, 3 \mathrm{H}), 2.10$ (m, 1H), $1.81(\mathrm{dd}, J=13.4,10.6 \mathrm{~Hz}, 1 \mathrm{H}), 1.53-1.25(\mathrm{~m}, 5 \mathrm{H}), 0.98$ (d, $J=6.6 \mathrm{~Hz}, 3 \mathrm{H}), 0.97(\mathrm{~d}, J$ $=6.7 \mathrm{~Hz}, 3 \mathrm{H}), 0.90(\mathrm{t}, J=7.2 \mathrm{~Hz}, 3 \mathrm{H})$, exchangeable protons not listed; ${ }^{13} \mathrm{C}$ NMR $(125 \mathrm{MHz}$, $\left.\mathrm{CDCl}_{3}\right) \delta 165.7,152.3,145.8,145.4,144.6,135.5,135.1,131.1,130.6,118.7,117.1,115.7$, $113.3,77.2,76.6,73.5,72.5,70.9,61.3,54.5,39.0,38.4,36.7,36.5,35.5,33.4,20.0,16.02$, 15.96, 14.2, 14.1; IR (neat) 3414 (br), 2961, 1710, 1612, $1150 \mathrm{~cm}^{-1}$; ESIMS calcd for $\mathrm{C}_{31} \mathrm{H}_{46} \mathrm{O}_{7} \mathrm{Na}\left(\mathrm{M}^{+}+\mathrm{Na}\right) 553.3$, found 553.5; optical rotation $[\alpha]_{\mathrm{D}}{ }^{27}-116^{\circ}\left(c 0.020, \mathrm{CH}_{2} \mathrm{Cl}_{2}\right)$.

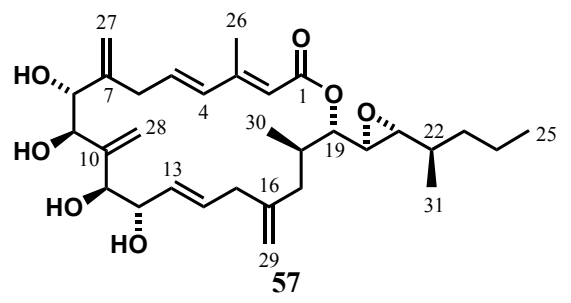

$\mathrm{R}_{f}=0.38$ (EtOAc); ${ }^{1} \mathrm{H}$ NMR $\left(500 \mathrm{MHz}, \mathrm{CDCl}_{3}\right) \delta 6.27(\mathrm{~d}, J=15.5 \mathrm{~Hz}, 1 \mathrm{H}), 6.08$ (ddd, $J=15.1$, 10.0, $5.0 \mathrm{~Hz}, 1 \mathrm{H}), 5.85$ (s, 1H), 5.68 (dddd, $J=15.4,7.1,7.1,1.2 \mathrm{~Hz}, 1 \mathrm{H}), 5.49$ (dd, $J=15.4,5.0$ $\mathrm{Hz}, 1 \mathrm{H}), 5.45$ (s, 1H), $5.34(\mathrm{~s}, 2 \mathrm{H}), 5.19(\mathrm{~s}, 1 \mathrm{H}), 4.85(\mathrm{~s}, 1 \mathrm{H}), 4.77$ (s, 1H), 4.65 (dd, J=6.7, 3.7 Hz, 1H), 4.62 (s br, 1H), 4.41 (s br, 1H), 4.20 (s br, 1H), 4.04 (s br, 1H), 3.20 (dd, J= 14.8, 4.4 Hz, 1H), $3.10(\mathrm{dd}, J=14.9,10.0,1 \mathrm{H}), 2.92(\mathrm{dd}, J=6.7,2.2 \mathrm{~Hz}, 1 \mathrm{H}), 2.75-2.72(\mathrm{~m}, 2 \mathrm{H}), 2.68$ $(\mathrm{dd}, J=7.5,2.2 \mathrm{~Hz}, 1 \mathrm{H}), 2.51(\mathrm{dd}, J=13.7,3.4 \mathrm{~Hz}, 1 \mathrm{H}), 2.27(\mathrm{~d}, J=0.9 \mathrm{~Hz}, 3 \mathrm{H}), 2.12(\mathrm{~m}, 1 \mathrm{H})$, $1.85(\mathrm{dd}, J=14.0,10.5 \mathrm{~Hz}, 1 \mathrm{H}), 1.53-1.24(\mathrm{~m}, 5 \mathrm{H}), 1.00(\mathrm{~d}, J=7.1 \mathrm{~Hz}, 3 \mathrm{H}), 0.97$ (d, $J=6.8 \mathrm{~Hz}$, $3 \mathrm{H}), 0.90(\mathrm{t}, J=7.2 \mathrm{~Hz}, 3 \mathrm{H})$, exchangeable protons not listed; ${ }^{13} \mathrm{C} \mathrm{NMR}\left(125 \mathrm{MHz}, \mathrm{CDCl}_{3}\right) \delta$ $165.9,152.8,147.7,145.1,144.8,136.5,134.8,131.0,130.4,118.9,115.6,114.4,113.2,76.5$, 75.7, 74.1, 72.4, 70.4, 61.2, 54.7, 39.1, 38.9, 36.7, 36.3, 35.4, 33.7, 20.0, 16.0, 15.8, 14.2, 13.8; IR (neat) 3424 (br), 2928, 1709, 1611, $1163 \mathrm{~cm}^{-1}$; ESIMS calcd for $\mathrm{C}_{31} \mathrm{H}_{46} \mathrm{O}_{7} \mathrm{Na}\left(\mathrm{M}^{+}+\mathrm{Na}\right)$ 553.3 , found 553.4; optical rotation $[\alpha]_{\mathrm{D}}^{27}+103^{\circ}\left(c 0.10, \mathrm{CH}_{2} \mathrm{Cl}_{2}\right)$. 